\title{
Diminished trkA receptor signaling reveals cholinergic-attentional vulnerability of aging
}

\author{
Vinay Parikh, ${ }^{1}$ William M. Howe, ${ }^{2}$ Ryan M. Welchko, ${ }^{3}$ Sean X. Naughton, ${ }^{1}$ Drew E. D'Amore, ${ }^{1}$ Daniel H. Han, ${ }^{1}$ \\ Monika Deo, ${ }^{3}$ David L. Turner ${ }^{3}$ and Martin Sarter ${ }^{2}$ \\ ${ }^{1}$ Department of Psychology and Neuroscience Program, Temple University, Philadelphia, PA, 19122, USA \\ ${ }^{2}$ Department of Psychology, University of Michigan, Ann Arbor, MI, 48109, USA \\ ${ }^{3}$ Molecular and Behavioral Neuroscience Institute, University of Michigan, Ann Arbor, MI, USA
}

Keywords: acetylcholine, cognition, rats, RNAi, trophic

\begin{abstract}
The cellular mechanisms underlying the exceptional vulnerability of the basal forebrain (BF) cholinergic neurons during pathological aging have remained elusive. Here we employed an adeno-associated viral vector-based RNA interference (AAV-RNAi) strategy to suppress the expression of tropomyosin-related kinase $A$ (trkA) receptors by cholinergic neurons in the nucleus basalis of Meynert/substantia innominata (nMB/SI) of adult and aged rats. Suppression of trkA receptor expression impaired attentional performance selectively in aged rats. Performance correlated with trkA levels in the nMB/SI. trkA knockdown neither affected nMB/SI cholinergic cell counts nor the decrease in cholinergic cell size observed in aged rats. However, trkA suppression augmented an age-related decrease in the density of cortical cholinergic processes and attenuated the capacity of cholinergic neurons to release acetylcholine (ACh). The capacity of cortical synapses to release ACh in vivo was also lower in aged/trkA-AAV-infused rats than in aged or young controls, and it correlated with their attentional performance. Furthermore, age-related increases in cortical proNGF and p75 receptor levels interacted with the vector-induced loss of trkA receptors to shift NGF signaling toward p75-mediated suppression of the cholinergic phenotype, thereby attenuating cholinergic function and impairing attentional performance. These effects model the abnormal trophic regulation of cholinergic neurons and cognitive impairments in patients with early Alzheimer's disease. This rat model is useful for identifying the mechanisms rendering aging cholinergic neurons vulnerable as well as for studying the neuropathological mechanisms that are triggered by disrupted trophic signaling.
\end{abstract}

\section{Introduction}

Cortically projecting cholinergic neurons arise from the nucleus basalis of Meynert/substantia innominata (nMB/SI) and constitute a major component of forebrain circuitry mediating cognitive, specifically attentional, processes and capacities (Everitt \& Robbins, 1997; Sarter \& Parikh, 2005; Parikh et al., 2007; Hasselmo \& Sarter, 2011). Decline in the regulation and subsequently the integrity of cholinergic neurons contributes to the severity of the cognitive symptoms in Alzheimer's disease (AD) and accelerates cytopathological processes yielding more generalized, telencephalic neurodegeneration (e.g. Mesulam, 2004; Mesulam et al., 2004).

Cholinergic neurons require nerve growth factor (NGF) signaling for development, maturation and function (Mobley et al., 1986; Hartikka \& Hefti, 1988; Kaplan \& Miller, 1997; Oosawa et al., 1999; Sofroniew et al., 2001). NGF exerts cellular effects through signaling via the high-affinity tropomyosin-related kinase A (trkA)

Correspondences: Dr V. Parikh, ${ }^{1}$ Department of Psychology and Neuroscience Program, as above.

E-mail: vinay.parikh@temple.edu

Dr M. Sarter, ${ }^{2}$ Department of Psychology, as above.

E-mail: msarter@umich.edu

Received 1 November 2012, accepted 6 November 2012 and the low-affinity p75 receptor. Although there are complex interactions between these receptors (e.g. Ceni et al., 2010), the available evidence suggests that trkA receptor stimulation primarily generates pro-survival signals and maintains cholinergic function ( $\mathrm{Li}$ et al., 1995; Fagan et al., 1997; Huang \& Reichardt, 2003). In contrast, stimulation of the p75 receptor preferably activates apoptotic mechanisms (Roux \& Barker, 2002; Chao, 2003; Schor, 2005; Volosin et al., 2006).

Abnormal trophic factor support of cholinergic neurons, specifically a reduction of trkA signaling, precedes the loss of cholinergic neurons in $\mathrm{AD}$ and is correlated with the cognitive decline in earlystage patients (Mufson et al., 1995, 2000; Counts et al., 2004; Counts \& Mufson, 2005; Ginsberg et al., 2006). Together with the evidence from studies on animals lacking trkA receptors throughout development (Fagan et al., 1997; Sanchez-Ortiz et al., 2012), evidence supports the hypothesis that loss of trkA receptors contributes to the degeneration of forebrain cholinergic neurons in AD. However, the mechanisms responsible for the detrimental interactions between aging processes and the impact of trkA dysfunction have remained less clear, specifically with regard to premorbid states when the cholinergic neurons appear to remain intact.

A valid rat model of the age-related vulnerability of nMB/SI neurons should reproduce the decline in trkA support and impair 
cholinergic and associated cognitive functions while sparing, at least transiently, the integrity of $\mathrm{nMB} / \mathrm{SI}$ cholinergic neurons as seen in patients with early-stage AD or mild cognitive impairments (references above). Aging in rats per se does not robustly affect the capacity of cholinergic neurons to support closely associated cognitive, specifically attentional, functions (reviewed by Sarter \& Bruno, 1998). Therefore, suppression of trkA receptors was hypothesized to serve as a 'second hit' to generate a model of age-related cholinergic decline. The experiments described below employed a viral vector-based RNA interference (RNAi) strategy to suppress the expression of trkA receptors by cholinergic neurons in the nMB/SI. Furthermore, both adult and aged rats were tested to determine the dependency of cholinergic function on trkA signaling while excluding the developmental impact of traditional trkA knockout models. The results demonstrate that TrkA suppression disrupts cortical cholinergic transmission and associated attentional capacities but does not affect the integrity of nMB/SI neurons. TrkA suppression produces cholinergic and cognitive decline in aged rats because the increased levels of proNGF and p75 receptors in aged rats interact with reduced trkA levels to suppress the cholinergic phenotype.

\section{Materials and methods}

\section{Plasmid construction}

To achieve trkA RNAi, we employed an expression vector (pUI4-GFP-SIBR) that utilizes an RNA polymerase II promoter, human ubiquitin $\mathrm{C}$ (Ubc) to express both short hairpin RNAs (shRNAs) and enhanced green fluorescent protein (GFP) from a single transcription unit. These shRNAs were generated in cells by endonuclease cleavage of a modular RNA structure, the SIBR (synthetic BIC-derived RNA) cassette, based upon the mouse miR-155 microRNA precursor and located in an intron of the GFP transcript (Chung et al., 2006; Taylor et al., 2008; Dickson et al., 2010). Synthetic DNA oligos encoding shRNA mRNA were designed to target the rat trkA receptor in accordance with functional characteristics proposed for the design of siRNAs (Reynolds et al., 2004) and were inserted into the SIBR cassette as previously described (Chung et al., 2006). Target sequences for shRNAs targeting trkA at nucleotides 403, 682, 1281 and 1455 (GenBank accession no. M85214) were: 5'-AUUCAGGUGACUGAGCCGAGGG-3', 5'-AAAAACGUCAUCCCCCACUUCC-3', 5'-UUCUUCUCCACUGGGUCUCUUG-3' and 5'-AUGAAGUGUAGGGACAUGGCCA-3'. A vector expressing a functional shRNA against firefly luciferase (luc) was used for experiments to control for potential non-selective effects of the RNAi vectors (Chung et al., 2006; Taylor et al., 2008).

\section{In vitro screening of plasmids}

Rat pheochromocytoma (PC12) cells were used to screen plasmids expressing trkA shRNAs for trkA suppression and differentiation. PC12 cells (CRL-1721.1; ATCC, Manassas, VA, USA) were maintained in F12K media supplemented with $10 \%$ horse serum, 5\% bovine serum, $50 \mathrm{U} / \mathrm{mL}$ penicillin and $50 \mu \mathrm{g} / \mathrm{mL}$ streptomycin sulfate. Cells were plated in cell culture dishes coated with ECL cell attachment matrix (EMD Millipore Co., Temecula, CA, USA) and subcultured every 5-7 days. Because the transfection efficiency in PC12 cells is usually low (25-30\%; see Results), transient puromycin-selection was adopted to screen plasmids for trkA protein suppression. Briefly, cells were grown in coated cell plates $(60 \mathrm{~mm})$ and after attaining 70-80\% confluency were transiently co-transfected with either pUI4-GFP-SIBR-trkA or pUI4-GFP-SIBR-luc
$(1.2 \mu \mathrm{g})$ and a US2 plasmid $(0.4 \mu \mathrm{g})$ encoding the puromycin-resistant gene (Chung et al., 2006; Taylor et al., 2008), using Lipofectamine 2000 (Invitrogen, Carlsbad, CA, USA). Transfected cells were treated with puromycin $(10 \mu \mathrm{g} / \mathrm{mL})$ for 3 days. Puromycin-resistant cells were selected, lysed and processed for Western blotting. Cell lysate proteins $(10 \mu \mathrm{g}$ per sample) were separated using SDS-PAGE followed by trkA immunoblot analysis (see Immunoblotting methods below). Five experiments per plasmid were performed and all samples were analysed in duplicate.

Morphometric analysis of NGF-differentiated PC12 cells was performed as described earlier (Das et al., 2004). Briefly, cells were plated on glass cover slips coated with ECL cell attachment matrix in 12-well plates at a density of 50000 cells per well. Following transfection with plasmids, the cells were incubated with complete F12 medium containing $100 \mathrm{ng} / \mathrm{mL}$ of recombinant NGF (Sigma, St Louis, MO, USA). The cells were fed every alternate day with NGF-containing media up to 5 days and then processed for imaging. Cells were fixed in $4 \%$ paraformaldehyde, rinsed in $0.01 \mathrm{M}$ PBS and mounted on slides with Prolong Gold (Invitrogen, Grand Island, NY, USA). Digitized images from 3 to 5 fields were obtained using a Leica DM4000B epifluorescent microscope (Leica Microsystems Inc., Buffalo Grove, IL, USA) and transfected cells were examined for differentiation and neurite outgrowth. Twenty-five GFP-positive cells were analysed for each experiment. Cells were classified as differentiated if the length of at least one neurite per cell was longer than the cell body. Neurite outgrowth analysis was performed by tracing the neurites using the NIH IMAGEJ plugin 'Neurite Tracer' (http://rsb.info.nih.gov/ij). The length of longest neurite per cell was used for analyses. Morphometric data are expressed as mean \pm SEM of four experiments from cultures prepared on separate days.

\section{Viral vectors}

The adeno-associated viral (AAV) SIBR vector plasmid pAAVSIBR was constructed on the basis of the pTR-UF5 vector (Peel et al., 1997). pTR-UF5 was digested with Bgl2 and sequences between the two inverted terminal repeats (ITRs) were replaced with the Ubc-driven shRNA/GFP/SV40 late polyadenylation (polyA) site transcription unit from pUI4-GFP-SIBR (Peel et al., 1997). A second transcription unit, located $3^{\prime}$ to the SV40 polyA site, expresses a puromycin resistance gene under the control of the mouse PGK promoter, followed by a bovine growth hormone polyadenylation site (Fig. 2A). The most effective siRNA construct, defined by suppression of $>70 \%$ trkA receptor expression in PC12 cells (above), was selected for in vivo studies. SIBR cassettes (see above) for the shRNA targeting trkA at position 403 or the luciferase control shRNA were inserted into pAAV-SIBR vector. Vectors were packaged using a serotype AAV2/1 helper free system (Gene Transfer Vector Core, University of Iowa, Iowa City, IA, USA). The packaged recombinant pAAV vectors are henceforth referred to as AAVtrkA and AAV-luc, respectively. The reproducibility of trkA knockdown produced by AAV-trkA shRNA was confirmed by assessing an additional recombinant AAV vector construct with the shRNA targeting trkA at base position 1455 (see Results).

\section{Animals}

Male Wistar rats aged 3 months (250-300 g) and 10-12 months (350-400 g; see Results for details of animal ages at the time of infusion) were procured from Charles River Laboratories (Malvern, PA, USA). The animals were housed individually in a 
temperature-/humidity-controlled environment with a 12-h dark/light cycle. Retired breeders were maintained in the animal housing facilities until they reached 22 months of age. At this point training of the operant sustained attention task (SAT) was initiated. Task training took place 6 days a week. Rats were water-deprived by restricting access to water to a 10-min period following each operant session (in addition to the water delivered as reward for correct responses; below). Water access was increased to $30 \mathrm{~min}$ on days when animals were not trained/tested. Food was available ad libitum. The experiments were conducted in accordance with the guidelines laid down by the NIH in the US regarding the care and use of animals for experimental procedures. All protocols were approved by the Institutional Biosafety Committee and the Institutional Animal Care and Use Committee at Temple University and the University of Michigan.

\section{Stereotaxic surgeries and viral vector infusions}

All animal surgeries were performed under aseptic conditions. Anesthesia was induced with isoflurane (4-5\%) using an anesthesia machine (Surgivet, Dublin, OH, USA). Rats were placed in a stereotaxic frame (Model 962; David Kopf Instruments, Tujunga, CA, USA) and anesthesia was maintained throughout the surgical procedure with $2-3 \%$ isoflurane using oxygen as a carrier gas at a flow rate of $0.6 \mathrm{~mL} / \mathrm{min}$. The body temperature of animals was maintained at $37{ }^{\circ} \mathrm{C}$ using isothermal deltaphase pad (Braintree Scientific, Braintree, MA, USA). For in vivo validation studies, young naö̈ve rats $(n=15)$ received unilateral infusions of AAV-trkA $\left(2.0 \mu \mathrm{L}\right.$; titer: $\left.2.8 \times 10^{13} \mathrm{vg} / \mathrm{mL}\right)$ into either the right or the left $\mathrm{nBM} / \mathrm{SI}$ region of the basal forebrain (AP: $-1.2 \mathrm{~mm}$, ML: $\pm 2.7 \mathrm{~mm}$ from bregma; DV: $-7.0 \mathrm{~mm}$ from dura; see Fig. $2 \mathrm{~A}$ ). Infusion of the control vector (AAV-luc, $2.0 \mu \mathrm{L}$; titer: $0.7 \times 10^{13} \mathrm{vg} / \mathrm{mL}$ ) into the contralateral hemisphere of the same animal served as within-animal control. Infusions were made using a $10-\mu \mathrm{L}$ Hamilton syringe and at a rate of $1.0 \mu \mathrm{L} / \mathrm{min}$; the needle remained in place for an additional $4 \mathrm{~min}$ following infusions. AAV-trkA and control vector infusions were counterbalanced across hemispheres. Animals were allowed to recover for 2, 4 or 8 weeks. Thereafter, brain sections were prepared for immunohistochemical examination of GFP/ChAT and trkA expression. An additional group of animals $(n=4)$ was used for immunoblotting experiments to determine cortical trkA expression levels following AAV-luc or AAV-trkA infusions. For behavioral studies (below), young and aged rats that reached criterion performance, received bilateral infusions of either AAV-trkA or AAV-luc vectors as described above.

\section{Sustained attention task (SAT)}

\section{Apparatus}

Behavioral training and testing took place in rat operant chambers (Med Associates Inc., St. Albans, VT, USA) equipped with two retractable levers, a houselight $(2.8 \mathrm{~W})$, a water dispenser, a 2900$\mathrm{Hz}$ sonalert tone generator and three panel lights (2.8 W each). Signal presentations, lever operations and reward delivery were controlled by an IBM 486 PC clone and MED-PC software (Med Associates).

\section{Behavioral training and testing procedures}

Animals were trained in SAT as described previously (Kozak et al., 2007; Demeter et al., 2008; St Peters et al., 2011). In brief, rats were initially trained to discriminate between signal (illumination of the central panel light for $1 \mathrm{~s}$ ) and non-signal (non-illumination) events. Two seconds following an event, both levers were extended and remained available for $4 \mathrm{~s}$ or until a lever press occurred. If no lever press occurred after $4 \mathrm{~s}$, an omission was registered and the intertrial interval (ITI; $12 \pm 3 \mathrm{~s}$ ) was reinstated. On signal trials, a left lever press was scored as a 'hit' and rewarded (0.02 $\mathrm{mL}$ water); depression of the right lever was considered an incorrect response and scored as a 'miss'. During non-signal trials, a right lever press was scored as a 'correct rejection' and reinforced, while a left lever press was considered an incorrect response and scored as a 'false alarm'. The animals were not rewarded for incorrect responses. The presentation of signal and non-signal trials was pseudo-randomized. Half of the animals in a group were trained with the reverse set of rules. After three consecutive days of $70 \%$ correct responses to signal and non-signal trials, animals progressed to the final phase of training during which the duration of signals was decreased to 25,50 or $500 \mathrm{~ms}$, house light was illuminated throughout the session, and the ITI was reduced to $9 \pm 3$ s. This final modification is a critical step in the acquisition of SAT as it requires the rats to constrain their behavior for continuous monitoring of the stimulus panel (for analyses of the attentional mechanisms taxed by this task see Demeter et al., 2008; Luck et al., 2012). Each behavioral session consisted of a pseudorandomized sequence of 81 signal (27 per signal duration) and 81 non-signal trials.

At the beginning of SAT training, animals were aged $2.84 \pm 0.07$ and $21.29 \pm 0.25$ months, respectively. AAV vectors were infused bilaterally into the $\mathrm{nBM} / \mathrm{SI}$ after the animals reached criterion performance of $>70 \%$ hits and correct rejections and $<20 \%$ omissions per session, for at least three consecutive sessions. Following a 3 - to 6-day post-surgery recovery period, animals resumed task practice and performance was monitored for 4 weeks. This time point was chosen based on results from our in vivo validation studies that suggested that maximum trkA knockdown is attained with AAV-mediated RNAi within 4 weeks. For the final analyses of the effects of trkA knockdown in young and aged rats, behavioral data from the last three sessions were averaged (see Results from details on the age of the animals and the time since infusion). After completion of behavioral studies, animals were prepared for either amperometric recordings or immunoblotting studies.

\section{Behavioral measures}

The numbers of hits (h), misses (m), correct rejections (cr), false alarms (fa) and omissions were recorded for the entire session and extracted post hoc for three equal blocks of 43 trials each to determine potential changes in performance across sessions. The relative number of hits $[\mathrm{h} /(\mathrm{h}+\mathrm{m})$; per signal duration], correct rejections $[\mathrm{cr} /(\mathrm{cr}+\mathrm{fa})]$ and omissions (omitted trials/162) were calculated and used for statistical analyses.

\section{In vivo amperometric recordings of evoked acetylcholine (ACh) release}

Ceramic-based, multi-site microelectrodes featuring four rectangular $(15 \times 333 \mu \mathrm{m})$ platinum recording sites arranged in side-by-side pairs (Quanteon, Nicholasville, KY, USA) were employed for in vivo amperometric recordings of evoked ACh release from prefrontal terminals. Choline-sensitive microelectrodes were prepared by immobilization of choline-oxidase (ChOase) enzyme to a pair of 
platinum channels and calibrated in vitro as described earlier (Parikh et al., 2008, 2010). The other pair of recording channels served as a sentinel control and was coated only with bovine serum albumin. All microelectrodes were electroplated with $m$-phenylenediamine prior to calibration. This step enhances the selectivity for choline by preventing access of potential electroactive interferents such as dopamine (DA), ascorbic acid (AA) and uric acid. Calibrated electrodes that met the following calibration criteria were subsequently used for in vivo recordings - choline sensitivity, $>3 \mathrm{pA} / \mu \mathrm{M}$; limit of detection (LOD), $<500 \mathrm{~nm}$ choline; selectivity ratio for choline to AA, > 80; linear response to increasing choline concentrations, $R^{2}>0.98$; electrode response to DA $(2 \mu \mathrm{M}),<3 \mathrm{pA}$ on all channels.

Viral vector-infused young and aged rats were prepared for in vivo amperomteric recordings. Animals were anesthetized with urethane $(1.25-1.5 \mathrm{~g} / \mathrm{kg}$; i.p.) and placed in a stereotaxic frame. The body temperature of animals was maintained at $37{ }^{\circ} \mathrm{C}$ using an isothermal deltaphase pad during the entire experimental session. Single-barrel glass capillaries $(1.0 \mathrm{~mm} \times 0.58 \mathrm{~mm}$, six in.; A-M systems) were pulled using a micropipette puller (Model \# 51210; Stoelting, Wood Dale, IL, USA) and then bumped against a glass slide to attain an inner tip diameter of approximately $15 \mu \mathrm{m}$. The micropipette was attached to the ceramic platform of the calibrated microelectrode and the tip was positioned between the lower and upper pair of recording sites. The spacing between the tip of the micropipette and the microelectrode was maintained at 70-100 $\mu \mathrm{m}$. The micropipette was loaded with one of the test solutions prior to microelectrode implantation. $\mathrm{An} \mathrm{Ag} / \mathrm{AgCl}$ reference electrode prepared from a miniature silver wire $(200 \mu \mathrm{m}$ diameter $)$ was implanted at a site remote from the recording area. The microelectrode/micropipette assembly was slowly lowered into the medial prefrontal cortex (AP: $+3.0 \mathrm{~mm}$; ML: $-0.7 \mathrm{~mm}$, and DV: $-3.0 \mathrm{~mm}$, measured from dura) using a microdrive (MO-10; Narishige International, East Meadow, NY, USA). Recordings were made at a frequency of $2 \mathrm{~Hz}$ and data were digitized using a FAST-16 recording system (Quanteon). Experiments began following stabilization of the baseline current for $60 \mathrm{~min}$. Depolarization-evoked ACh release was induced by applying a brief pulse [2-10 p.s.i. (13.8-69 $\mathrm{kPa}) ; 0.8-2 \mathrm{~s}$ ] of potassium ( $\mathrm{KCl} 70 \mathrm{~mm} ; 200 \mathrm{~nL}$ ) from the micropipette using PTFE tubing connected to a pressure ejection system (Picospritzer NPI; ALA Scientific Instruments, Farmingdale, NY, USA). At the end of recording sessions, animals were perfused and brains were isolated either for immunohistochemistry (below) or for Nissl-staining to tract placement of electrodes.

Potassium-evoked choline signal responses were analysed with respect to peak signal amplitudes (Parikh \& Sarter, 2006). Currents recorded via enzyme-coated sites were self-referenced by subtracting currents recorded on sentinel sites (Parikh et al., 2008, 2010) in cases when background noise levels on enzyme-coated channels exceeded $10 \mathrm{pA}$ or pressure-ejection artifacts occurred. The averages of three signal amplitudes per animal were used for statistical analysis.

\section{Quantitative immunohistochemistry and unbiased stereology}

Anesthetized animals were transcardially perfused with $100 \mathrm{~mL}$ of ice-cold heparinized saline followed by $300 \mathrm{~mL}$ of $4 \%$ paraformaldehyde (in $0.1 \mathrm{M}$ PBS; pH 7.4). Brains were removed, post-fixed overnight and then stored in $30 \%$ sucrose in $0.1 \mathrm{M}$ PBS for $72 \mathrm{~h}$. Coronal sections $(50 \mu \mathrm{m})$ were sliced on a freezing microtome (CM2000R; Leica, Chantilly, VA, USA) and stored in cryoprotectant solution (15\% glucose, $30 \%$ ethylene glycol and $0.04 \%$ sodium azide in $0.05 \mathrm{M}$ PBS) at $-20{ }^{\circ} \mathrm{C}$ until further processing. Immunostaining was performed on free floating sections.

\section{In vivo transduction efficiency of $A A V$ vectors and efficacy for trkA} knockdown

Randomly sampled serial sections at the level of the nBM/SI (0.84-2.16 mm posterior to bregma) were processed for GFP/ ChAT fluorescence immunostaining. After thawing, the sections were washed twice in $0.05 \mathrm{M}$ Tris-buffered saline (TBS) for $5 \mathrm{~min}$, and blocked in $10 \%$ donkey serum for $1 \mathrm{~h}$ at room temperature. The sections were then incubated with goat anti-ChAT antibody (EMD Millipore Co.) diluted to $1: 200$ in $0.05 \mathrm{M}$ TBS containing $1 \%$ donkey serum overnight at $4{ }^{\circ} \mathrm{C}$ with constant shaking. The following day, sections were washed in 0.05 м TBS containing $1 \%$ triton $\mathrm{X}-100$ (wash buffer) three times for $5 \mathrm{~min}$ and incubated with $1: 250$ diluted rhodamine-conjugated donkey anti-goat antibody (Jackson ImmunoResearch Laboratories Inc., Westgrove, PA, USA) for $2 \mathrm{~h}$. After four 10-min washes in wash buffer, sections were mounted on gelatin-coated slides and coverslipped with Prolong-Gold anti-fade solution (Invitrogen). Virus spread was determined on the basis of GFP expression throughout the rostro-caudal nBM/SI.

The efficacy of AAV-trkA vectors to knockdown basal forebrain (BF) trkA receptors was determined by conducting trkA immunostaining on parallel sections from the same animals used for GFP/ ChAT double-immunostaining. Briefly, sections were blocked in $10 \%$ goat serum and incubated with rabbit anti-trkA antibody (1: 1000 dilution) overnight. Sections were washed and incubated in $1: 50$ diluted biotinylated goat anti-rabbit IgG (EMD Millipore Co.) for $2 \mathrm{~h}$ followed by streptavidin-horseradish peroxidase (HRP; $1: 1000$ dilution) for 45 min with constant shaking. Sections were washed in $0.05 \mathrm{M}$ TBS and staining was developed with 3-3'-diaminobenzidine in the presence of $0.01 \%$ nickel chloride (DAB-Ni). Stained sections were mounted onto gelatin-coated slides and air dried. Slides were then dehydrated and coverslipped with DPX. Control sections were processed in the absence of either the primary or secondary antibody.

All sections were analysed using a Leica fluorescent microscope equipped with a DFC 425C digital camera and Leica Application Suite image acquisition system (Leica Microsystems Inc.). To determine transduction efficiency, sections were evaluated for the expression of GFP and ChAT. Images were captured from both green and red filter simultaneously using the Image Overlay Module at $200 \times$ magnification and two representative fields in each hemisphere/section were analysed for GFP/ChAT double-immunostaining. Fifty infected neurons in the $\mathrm{nBM} / \mathrm{SI}$ region per hemisphere from a total of three sections per animal (100 neurons/animal) were analysed. The proportion of GFP-positive neurons that colocalized with ChAT-expressing neurons was determined. Values per hemisphere were used to indicate the efficacy of the vectors and values averaged over both hemispheres indicated the extent of virus infection. For analysis of nBM/SI trkA expression, images were acquired at $400 \times$ magnification in the light microscope mode and processed for quantitative image analysis using NIH IMAGEJ analysis software as described earlier (Parikh \& Sarter, 2006). Digitized images were processed in binary mode and threshold levels were adjusted to enhance the visibility of cell bodies. The density of trkA receptors was expressed as percentage of trkA-positive pixels in the analysed area $(325 \times 245 \mu \mathrm{m})$. Averaged trkA densities were based on analyses from three sections per animal. The two hemispheres were analysed separately. 
Stereological cholinergic cell counts, cholinergic morphology and presynaptic ChAT density in AAV-luc/trkA-infused young and aged rats

For quantification of nBM/SI cholinergic cells, serial sections from $\mathrm{nBM} / \mathrm{SI}$ collected at $500-\mu \mathrm{m}$ intervals, from 0.84 to $2.64 \mathrm{~mm}$ posterior to bregma (plates 40-55; Paxinos \& Watson, 2007) were processed for ChAT-immunohistochemistry. An average of five sections $(50 \mu \mathrm{m})$ per animal, representing $\mathrm{nBM} / \mathrm{SI}$ region of $\mathrm{BF}$ along the rostral-caudal axis, were processed for ChAT immunostaining. To determine presynaptic ChAT density, serial coronal sections $(50 \mu \mathrm{m})$ from the prefrontal cortex were used. Free-floating sections were incubated with goat anti-ChAT antibody (1: 100 dilution; EMD Millipore Co.) and peroxidase staining was developed using HRP-conjugated donkey anti-goat (1:1000 for cell bodies and $1: 200$ for terminals) and DAB.

Unbiased stereological assessment of ChAT-positive cell counts was performed using the optical fractionator counting procedure (West et al., 1991). Sections were viewed under a Nikon Optiphot 2 light microscope equipped with a motorized stage and an Optronics MicroFire color digital camera. Boundaries of cholinergic nuclei in the anterior and posterior SI, and nBM (between exterior globus pallidus, internal capsule and optic tract) region were delineated on whole brain sections under low magnification $(4 \times$ objective) using NeUrolucida software (MBF Bioscience, Williston, VT, USA). Cholinergic nuclei in the horizontal limb and diagonal band of Broca were not included in these analyses. ChAT-immunopositive cells were counted with a $60 \times$ oil immersion objective $(\mathrm{NA}=1.4)$ in randomly placed counting frames $(50 \times 50 \mu \mathrm{m})$ on a counting grid $(190 \times 130 \mu \mathrm{m})$ using StereoInvestigator software (MBF Bioscience). Sampling was done with an optical dissector $(18 \mu \mathrm{m})$ with an average mounting thickness of $25 \mu \mathrm{m}$. All counting was performed by an observer (D.H.H.) blind to the vector infusions and to the age of the animals. The average Gunderson coefficient of error (CE) was $<0.09$.

Morphometric and quantitative analysis of cholinergic neurons involved measurement of cell size (cross-sectional area of soma) and ChAT densities using NIH IMAGEJ. Images from the right hemisphere were captured at $400 \times$ magnification to analyse ChAT-positive cells. ChAT expression was expressed as the percentage of ChAT-positive pixels in the analysed area $(325 \times 245 \mu \mathrm{m})$. Averaged ChAT densities were based on the analyses from three sections per animal. Mean cross-sectional area was calculated for a random selection of cholinergic cells in the nBM/SI region. Digitized images were processed in binary mode and threshold levels were adjusted to enhance the visibility of cell bodies uniformly among all sections with the help of a macro created in the software. To avoid cell size bias, only cells with a visible nucleus in the plane of section were analysed. Cell borders were identified manually by using the Analyze Menu in ImageJ. The area of the cell was automatically calculated by the program based on the length (in pixel units) and area (in square pixel units) of the userdefined border. Cells without apparent nuclei were excluded from the analysis. Cross-sectional area was converted from pixels ${ }^{2}$ to $\mu \mathrm{m}^{2}$ by scaling appropriately for $400 \times$ magnification (12.09 pixels $/ \mu \mathrm{m})$. Fifty cells were analysed from three sections per animal. Images captured for the analysis of cross-sectional area were later processed to examine changes in BF ChAT expression. The procedure for analysis of ChAT immunoreactivity was similar to trkA (described above).

Prefrontal ChAT-immunoreactive fiber density was analysed using a grid counting procedure as described earlier (St Peters et al.,
2011). Briefly, images from were captured with a $40 \times$ objective along three rostralcaudal planes (AP $3.2-2.7 \mathrm{~mm}$ anterior to bregma). ChAT-immunoreactive fibers were quantified in cortical layers III/V of the prelimbic region. Threshold levels were uniformly adjusted across all sections to maximize visualization of ChAT-ir fibers using a macro created in Adobe Photoshop CS4 (Adobe Systems Inc., San Jose, CA, USA). Fiber counts were made in an area of $200 \times 200 \mu \mathrm{m}$ using $50-\mu \mathrm{m}$ grids. Average counts were based on three sections per region per animal.

\section{$B F$ trkA and $p 75$ receptors in young and aged rats}

To determine the effects of age and trkA knockdown on BF NGF receptor expression, sections were also processed for BF trkA and p75 immunohistochemistry. All staining steps were similar as described above for trkA staining except that the primary antibody used to detect p75 receptors was a rabbit anti-p75 antibody (Covance Inc., Princeton, NJ, USA). Moreover, DAB was used to develop staining for p75 receptors instead of DAB-Ni. Sections were analysed for stained pixels in a given area (see above).

\section{Immunoblotting}

Animals were decapitated and prefrontal cortices were isolated. For in vivo validation experiments, tissues from each hemisphere were processed separately to determine the effects BF AAV-luc or AAVtrkA infusions on trkA expression. Tissues from both hemispheres were pooled together in the remaining experiments. Samples were homogenized in a glass tissue grinder using $1 \mathrm{~mL}$ of ice-cold extraction buffer (50 mm HEPES NaOH, pH 7.4, 0.32 м sucrose, $5 \mathrm{~mm}$ $\mathrm{MgCl}_{2}, 0.2$ mm EDTA, 0.2 mm EGTA, 1\% Triton X-100 and protease inhibitor cocktail). The homogenates were kept on ice for $30 \mathrm{~min}$ following which they were centrifuged at $13000 \mathrm{~g}$ for $15 \mathrm{~min}$ to obtain tissue lysates. Supernatants were stored at $-80{ }^{\circ} \mathrm{C}$ until analysed. Protein concentrations were determined by using a modified Lowry Protein Assay (Pierce, Rockford, IL, USA). Equal quantities $(25 \mu \mathrm{g})$ of protein were subjected to immunoblot analysis and each sample was assayed in duplicate. Proteins were separated on $4-15 \%$ Tris- $\mathrm{HCl}$ polyacrylamide gels and transferred to PVDF membranes. Following blocking in 5\% non-fat dry milk, membranes were incubated with primary antibodies (EMD Millipore Co. - rabbit anti-trkA, rabbit anti-proNGF; Covance Inc. - rabbit anti-p75) overnight on an orbital shaker at $4{ }^{\circ} \mathrm{C}$. Membranes were washed with $0.01 \mathrm{M}$ PBS containing $0.1 \%$ Tween and incubated with HRPconjugated sheep anti-rabbit antibody (GE Healthcare Biosciences, Pittsburgh, PA, USA). Blots were visualized for chemiluminescence detection using ECL Advance system (GE Healthcare) and images were captured using Molecular Imager Chemidoc EQ system (BioRad, Hercules, CA, USA). All membranes were stripped in Restore Plus buffer (Pierce) and reprobed with mouse anti- $\beta$-tubulin antibody to detect $\beta$-tubulin that served as a gel-loading control. Densitometric analysis was performed by calculating the integrated pixel densities using NIH IMAGEJ software. Blot densities of trkA, p75 and proNGF were normalized to the levels of $\beta$-tubulin-immunoreactive bands for each sample analysed.

\section{NGF ELISA}

NGF levels in prefrontal cortex were quantified using a rat NGF ELISA kit as per recommendations from the manufacturer (Promega, Madison, WI, USA). All lysates were analysed in duplicate and the data were expressed as $\mathrm{pg} / \mathrm{mg}$ protein. 


\section{Statistical analyses}

Statistical analyses were performed using SPSS/PC+ V17.0 (IBMSPSS, Armonk, NY, USA). For in vitro studies, the effects of SIBR plasmids on transfection efficiency, trkA immunoreactivity and neurite outgrowth were analysed using one-way ANOvAs followed by post-hoc comparisons using a Fisher's least significant difference (LSD) test. Analyses for in vivo validation studies employed mixedfactor ANOvAs to examine the effects of viral manipulation (two levels) and post-infusion time points (three levels) on infection efficiency and efficacy of trkA silencing. Unpaired $t$ tests were applied for multiple comparisons. Paired $t$ comparisons were conducted to analyse hemispheric differences for infection efficiency and trkA expression. For the behavioral data, the repeated-measures task block (three levels) and signal duration (three levels) were analysed with linear mixed models including viral manipulation (two levels) and age (two levels) as between-subjects variables. Covariance structures were selected based upon Akaike's Information Criterion, and depending upon the model employed, degrees of freedom were not always integers (Verbeke \& Molenberghs, 2009). Where appropriate, one-way ANOVAs and Fisher's LSD test were used for post hoc comparisons to determine the source of interactions. The effects of age and viral manipulations on cortical ACh release, ChAT stereology, cross-sectional area of cholinergic neurons, ChAT fiber densities, NGF/proNGF levels and NGF receptor densities were analysed using mixed-design ANOVAs. In cases of violation of the sphericity assumption, Huyhn-Feldt-corrected $F$-values, along with uncorrected degrees of freedom, are given. Post hoc comparisons between groups were conducted either using LSD or planned $t$ tests. Exact $P$-values are reported (Greenwald et al., 1996).

\section{Results}

\section{In vitro screening of SIBR plasmids expressing trkA shRNAs}

UI4-SIBR-GFP plasmids expressing four different shRNAs targeting rat trkA were screened in $\mathrm{PC} 12$ cells expressing trkA. Because of the lower transfection efficiency of PC12 cells, it was not possible to quantify trkA suppression using immunofluorescence. Therefore, we determined trkA expression from puromycin-selected PC12 cells using Western blotting, conducted 3 days after transfection (see Materials and methods). trkA expression levels from cells transfected with empty pUI4-GFP-SIBR plasmid (not expressing an shRNA) did not differ from the pUI4-GFP-SIBR-luc shRNA plasmid ( $t_{6}=0.60, P=0.56$ ), indicating the absence of non-selective effects of the pUI4-GFP-SIBR-luc control shRNA plasmid. trkA expression in cell lysates differed significantly across plasmid treatment groups $\left(F_{4,20}=20.66, P<0.001\right)$. All four trkA shRNA constructs $(403$, $682,1281$ and 1455$)$ reduced trkA receptor expression in PC12 cells (LSD - all $P<0.001$ vs. luc). The band pixel densities, normalized to $\beta$-tubulin, ranged from $39.32 \pm 7.87$ to $54.80 \pm 7.65$ (luc control: $135.26 \pm 10.13)$. The most efficacious trkA knockdown was generated by the plasmid expressing shRNA targeting the 403-bp position (Fig. 1A and B).

Neurite outgrowth analysis was conducted to determine whether reduction in trkA receptors produced by plasmid-based RNAi impaired growth and differentiation of PC12 cells. The effects of SIBR plasmids expressing trkA shRNAs and luc shRNA on cellular differentiation and neurite length, following a 5-day exposure to NGF (100 ng), are shown in Fig. 1C-E). NGF induced differentiation in $77 \pm 3 \%$ of the cells transfected with the control plasmid. Cells transfected with trkA plasmids exhibited significantly lower levels of differentiation, depending on the individual plasmid $\left(28 \pm 2-34 \pm 4 \% ; F_{4,15}=33.17, P<0.001 ;\right.$ LSD - all $P<0.001$ vs. luc). The length of the longest neurite was also reduced in NGFdifferentiated cells treated with trkA plasmids as compared with SIBR_luc plasmid $\left(F_{4,15}=73.56, P<0.001 ;\right.$ LSD - all $P<0.001$ vs. luc). Consistent with trkA expression analyses, these morphological analyses of NGF-reeated PC12 cells confirmed the greatest efficacy by the 403-bp-targeting shRNA.

\section{TrkA receptor knockdown using recombinant $A A V$ vectors in vivo}

\section{Infection space and cholinergic targeting}

Pilot experiments determined that infusions of $2.0 \mu \mathrm{L}$ per hemisphere of AAV vectors achieved maximal infection of the $\mathrm{nBM} / \mathrm{SI}$
A

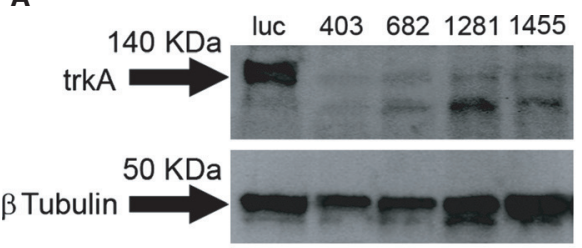

B

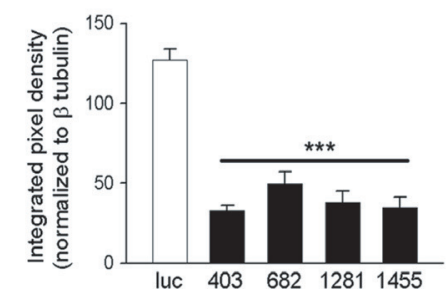

C

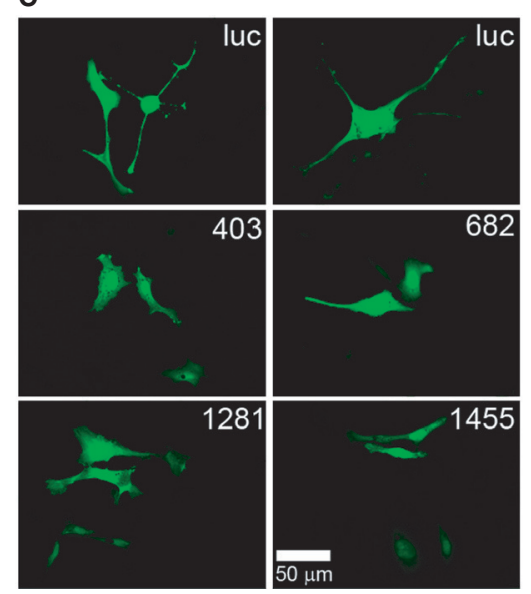

D
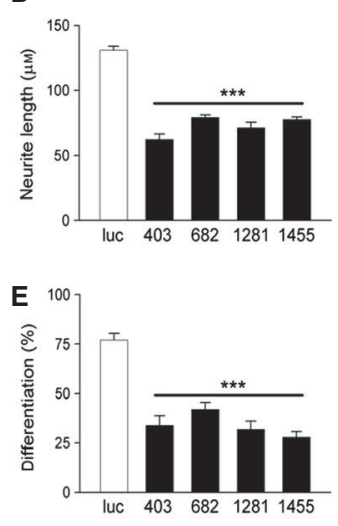

FIG. 1. In vitro screening of SIBR plasmids. (A) Immunoblots depicting trkA expression in puromycin-selected PC12 cells transfected with UI4-SIBR-GFP plasmids expressing trkA or control shRNAs. trkA was detected as a 140-kDa band by Western blot. (B) All four shRNA constructs significantly attenuated trkA receptor expression in PC12 cells $(* * * P<0.001$; mean $\pm \mathrm{SEM})$. (C) Representative images of GFP-expressing, transfected PC12 cells following NGF differentiation. Compared with neurite outgrowth following transfection with the functional control (luciferase; luc) plasmid, morphometric analysis revealed a marked decline in neurite length (D; longest neurite) and percentage differentiation (E) as a result of transfection with SIBR-trkA plasmids (all $P<0.001$ vs. luc) and thus impaired NGF signaling due to reduced trkA. 

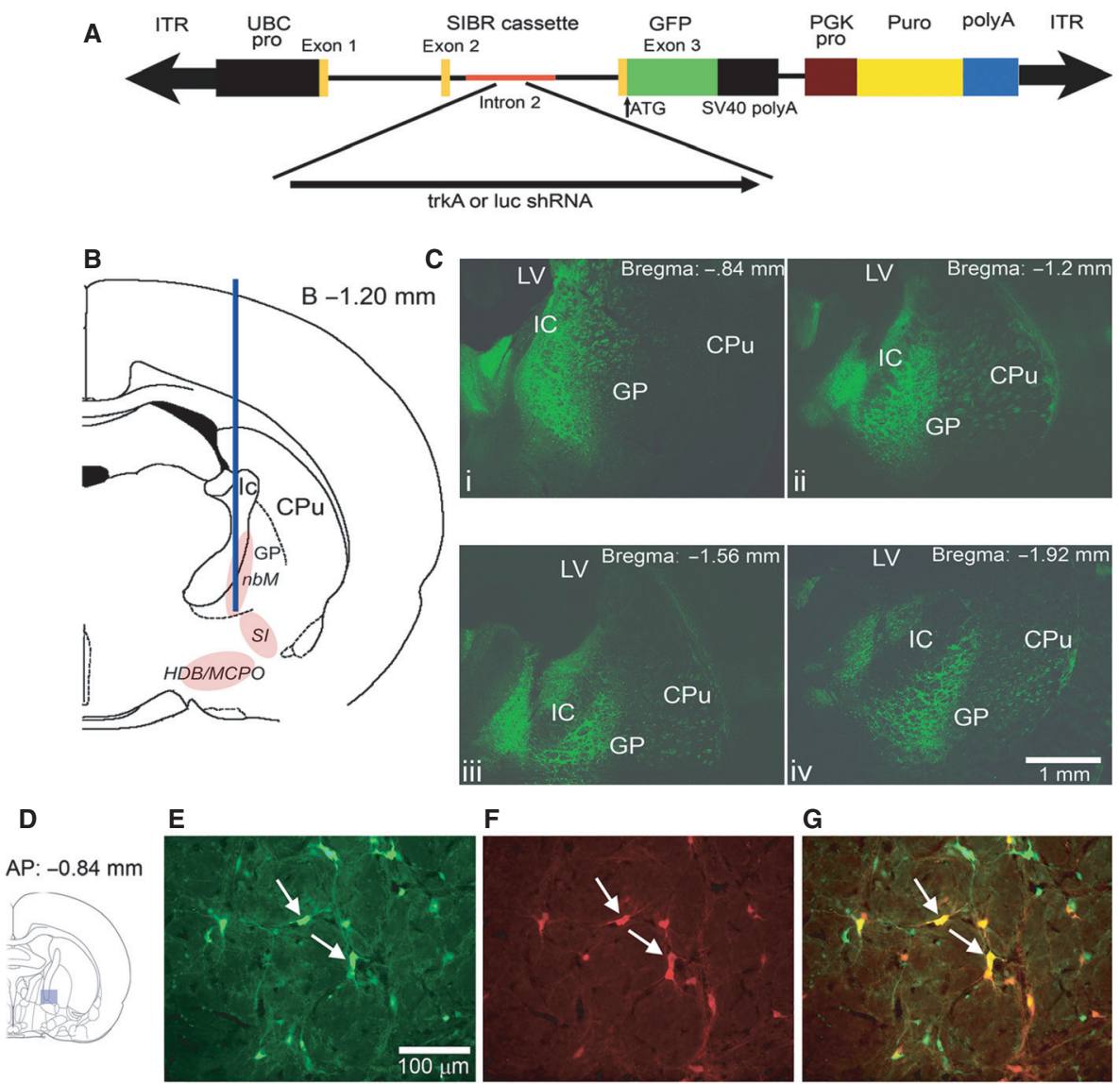

G
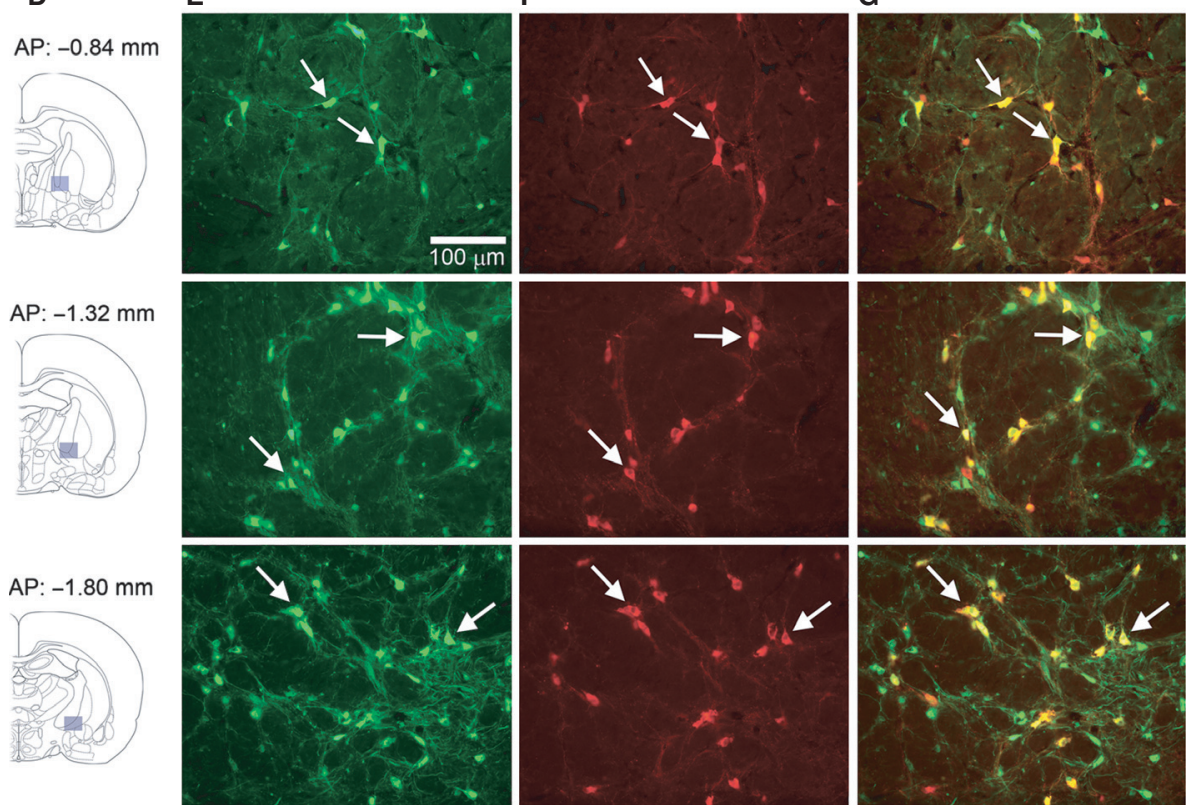

FIG. 2. In vivo transduction efficiency and cholinergic targeting of AAV vectors. (A) AAV-SIBR vector construct depicting UBC promoter-driven expression of an intronic microRNA-based shRNA (SIBR cassette) and a GFP reporter gene from the same transcript. A separate transcript expresses a puromycin resistance gene (depiction not to scale). (B) Coronal section depicting cannula placements for AAV vector infusions for in vivo validation studies. Animals received infusions of the AAV vector expressing trkA shRNA (AAV-trkA) into the nBM/SI region of one hemisphere and of the functional control AAV vector targeting inert gene luciferase (AAV-luc) into the contralateral hemisphere. The pink-shaded clouds depict the location of major cholinergic cell groups along the medial wall of the globus pallidus (GP), that is the nucleus basalis of Meynert (nBM), ventral to the GP, i.e. the substantia innominata (SI) and, more ventrally, the horizontal nucleus of the diagonal band and the magnocellular preoptic region (HDB/MCPO; IC, internal capsule; CPu, caudate-putamen). (C) GFP-expressing, AAV-luc infection space in the basal forebrain, including nBM in a series of coronal sections (from Bregma -0.84 to $-1.92 \mathrm{~mm}$; LV, lateral ventricle). In vivo transduction efficiency of AAV vectors was determined by GFP/ChAT double-immunohistochemistry. (D) Coronal sections depicting sampled areas in the $\mathrm{nBM} / \mathrm{SI}$ region to examine colocalization of GFP and ChAT (4 weeks post-infusion). Expression of GFP (column E) in cholinergic neurons (ChAT; column F) infected in vivo with a control vector 4 weeks post-infusion. (G) Merged images showing colocalization of GFP and ChAT in infected BF neurons (see arrowheads for double-labeled neurons). On average, $78 \pm 6 \%$ of the cholinergic neurons in the $\mathrm{nbM} / \mathrm{SI}$ region were infected.

region. The objective of these experiments was to knockdown trkA receptors expressed specifically by corticopetal cholinergic neurons originating from the nbM/SI region. Figure $2 \mathrm{C}$ illustrates the extent of GFP expression following infusion of the AAV-luc vector into the $\mathrm{nBM} / \mathrm{SI}$ region. Stereotaxic infusions of AAV vectors produced a total virus spread extending over $1.8 \mathrm{~mm}$ along the rostral-caudal axis but spared hippocampally projecting cholinergic neurons in the medial septum and the vertical diagonal band of Broca. Quantitative analyses revealed coexpression of GFP and ChAT (Fig. 2D-G) at all three post-infusion time points $(2,4$ and 8 weeks). However, GFP/ChAT colocalization differed across these time points $\left(F_{2,8}=5.99, \quad P=0.02\right)$, with significantly high 
coexpression rates after 4 weeks $(78 \pm 6 \%)$ when compared with 2 weeks post-infusion $\left(52 \pm 5 \% ; t_{6}=2.82, P=0.03\right)$. GFP/ChAT coexpression levels did not differ between 4 and 8 weeks postinfusion $\left(t_{6}=0.19, P=0.85\right)$. Infection efficiency differed neither between the two hemispheres $\left(t_{10}=1.31, P=0.22\right)$ nor between the control and the 403-bp-targeting trkA vector $\left(t_{10}=1.57\right.$, $P=0.15)$.

\section{Reduced expression of trkA receptors}

Figure $3 \mathrm{~A}-\mathrm{C}$ depicts trkA receptor expression in the nBM/SI following AAV-trkA or functional control vector infusions. AAV-trkA produced a robust reduction in $\mathrm{nMB} / \mathrm{SI}$ trkA expression $\left(F_{1,16}=122.98, P<0.001\right)$. Time-course analysis of trkA receptor levels indicated more effective suppression after 4 and 8 weeks when compared with 2 weeks post-infusion (time: $F_{2,16}=5.35$, $P=0.01$; time $\times$ group: $\left.F_{2,16}=3.72, P=0.04\right)$. Multiple post-hoc comparisons indicated lower trkA levels at all time points after infusions of AAV-trkA (all $P<0.01$ ) but lower levels after 4 weeks when compared with 2 weeks post-infusion $\left(t_{6}=4.73, P=0.003\right)$ and no difference between the two later time points. These findings are consistent with earlier studies that show AAV vectors require more than 2 weeks to show maximal gene transduction (Hommel et al., 2003; Koornneef et al., 2011). Furthermore, the AAV-trkA construct was specific for trkA receptors and did not affect the expression of other neurotrophin receptors e.g. trkB (see Supporting Information).

We also assessed the effects of AAV vector-based RNAi on cortical trkA receptor levels. As expected, immunoblot data indicated a significant reduction in trkA receptor densities following AAV-trkA infusions vs. control infusions [control: $35.66 \pm 2.10$ (arbitrary dimension; normalized trkA density); AAV-trkA: $19.98 \pm 1.53$; $t_{6}=3.91, P=0.008$; Fig. 3D).

To confirm whether trkA reduction in vivo is reproducible with different shRNAs, suppression of trkA receptors was compared for AAV vectors targeting two distinct sequences of trkA (403 and 1455 bp) 4 weeks post-infusion. Compared with the effects of the control vector, infusion of the alternate AAV-trkA (1455) vector likewise reduced $\mathrm{BF}$ trkA expression [1455: $0.62 \pm 0.11$ (arbitrary dimension; BF trkA pixel density); AAV-luc: $1.87 \pm 0.24$; $\left.t_{6}=3.95, P=0.008\right]$. Residual trkA levels did not differ between the two active constructs $\left(t_{7}=1.49, P=0.18\right)$. These findings indicate that both constructs similarly attenuated trkA expression. Finally, we acknowledge that these AAV vectors transfected cholinergic as well as non-cholinergic neurons in the infusion area; however, given that in this region virtually all trkA-positive neurons co-express ChAT (Sobreviela et al., 1994), infection of non-cholinergic BF neurons with an RNAi vector that attenuates the trkA receptor is not expected to impact the function of these neurons.

\section{TrkA knockdown impairs hit rate in aged, but not young, rats}

The results of the first series of experiments validated the efficacy of an AAV-SIBR vector for attenuating trkA receptor expression in $\mathrm{nMB} / \mathrm{SI}$ cholinergic neurons. We next used these vectors to determine effects on SAT performance. SAT performance depends on the integrity of the cholinergic system (McGaughy et al., 1996) and is mediated via increases in cholinergic neurotransmission (Apparsundaram et al., 2005; St Peters et al., 2011). Specifically, hits - but not misses - evoke brief cholinergic release events (Parikh et al., 2007). We hypothesized that if the loss of trkA receptors results in
A

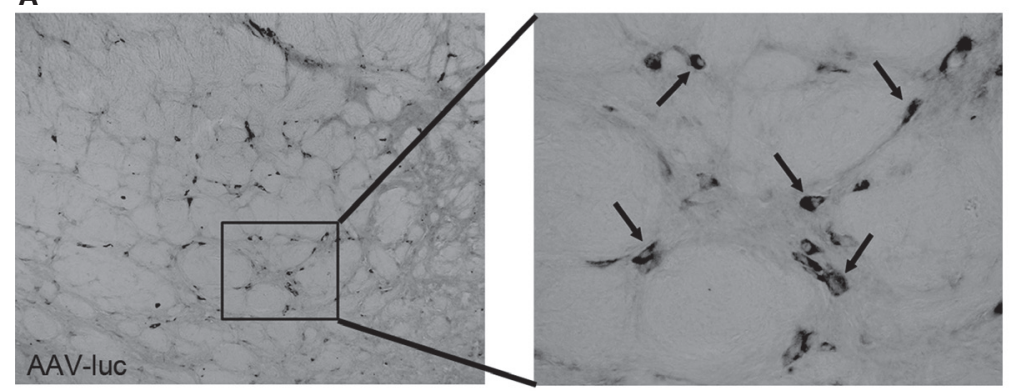

B

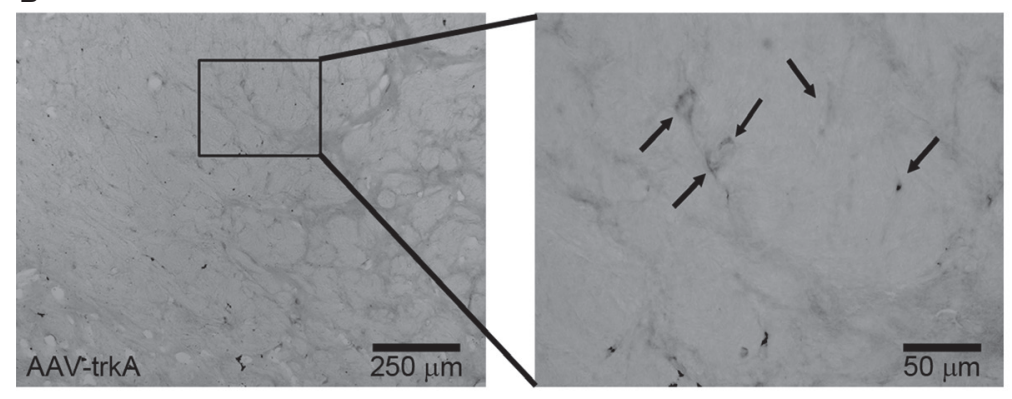

C

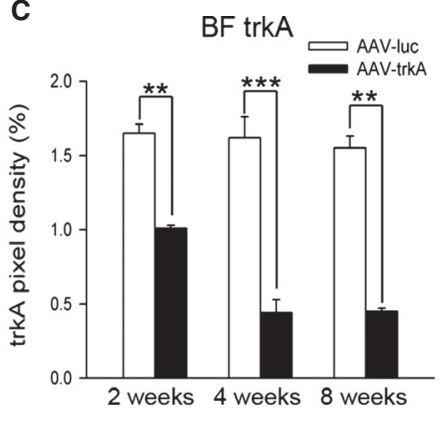

D

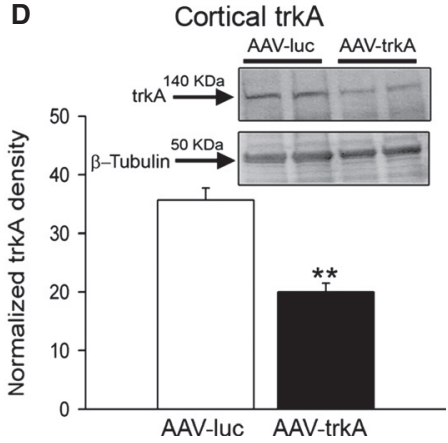

FIG. 3. TrkA receptor knockdown in the basal forebrain (BF) cholinergic projection system using AAV-mediated RNAi. Representative images from sections depicting trkA immunoreactivity in the nMB region of rats after 4 weeks of infusion of either the AAV-luc (A) or the AAV-trkA (B) vector (boxed areas are shown at higher magnification on the right; arrows point to trkA-immunopositive neurons). (C) AAV-trkA infusions produced a robust reduction in the cell pixel density of BF trkA-immunopositive neurons at all time points with maximum reduction in trkA levels observed 4 weeks post-AAV-trkA infusion (see Results). (D) TrkA receptor densities in homogenates prepared from prefrontal tissue were determined using immunoblotting. Immunoblot analysis reveal a robust decline in cortical trkA expression in BF trkA knockdown animals. Unpaired $t$-tests: ${ }^{* *} P<0.01,{ }^{* * *} P<0.001$ vs. AAV-luc. 
a decline in cholinergic function, SAT performance will be impaired and this effect will be correlated with measures of cholinergic activity.

Rats were aged $2.84 \pm 0.07$ and $21.29 \pm 0.25$ months at the beginning of SAT training. Aged rats required more training to reach the final performance criterion (defined in Materials and methods; $47.73 \pm 3.94$ vs. $103.20 \pm 5.73$ days; $\left.t_{32}=7.43, P<0.001\right)$. Consistent with the observation that a 4 -week post-infusion period suffices to generate maximum trkA suppression (above), the three final sessions of SAT performance used for data analysis were obtained $33.03 \pm 0.96$ days after infusion of the vectors (active or control; see Materials and methods for surgery and post-infusion SAT training). Data from final sessions were obtained from rats aged $6.11 \pm 0.26$ and $25.90 \pm 0.28$ months, respectively. This age of the older rats is considered equivalent to the human post-reproductive period (60-70 years) and precedes the onset of accelerating mortality of this strain (Nistiar et al., 2012).

Prior to receiving bilateral microinjections of either the AAV-luc or the AAV-trkA vector, SAT performance did not differ between young and aged animals nor between animals within the age groups that were designated for AAV-luc or AAV-trkA infusions, respectively (all measures of performance including omissions; all $P>0.29)$. For all animals, performance on signal trials was stimulus-duration-dependent $\left(F_{2,108}=145.89, P<0.001\right)$, with the highest number of hits to longest signals [500 ms: $87.53 \pm 1.41 \%$ hits (mean \pm SEM); 50 ms: $62.64 \pm 2.09 \% ; 25$ ms: $42.17 \pm 1.87 \%$ ]. Animals correctly rejected $79.05 \pm 1.46 \%$ of non-signal events and omitted $3.73 \pm 0.99 \%$ of all trials. Following the post-surgery recovery period, animals returned to daily SAT practice.

Infusion of the trkA-AAV vector impaired SAT performance, specifically by reducing the relative number of hits, beginning during week 3 post-infusion (Fig. 4A). Analysis of asymptotic performance was based on performance data from the last three sessions prior to tissue harvesting or the electrochemical recordings (below). A significant interaction between the effects of age (young, aged), vector (trkA-AAV vs. AAV-luc) and signal duration on hits $\left(F_{2,222.76}=4.13, P=0.017\right.$; Fig. $\left.4 \mathrm{~B}\right)$ reflected that the hit rate of aged rats was lower than that in the other three groups. There were also main effects of vector [AAV-luc: $65.46 \pm 1.27 \%$; AAV-trkA: $55.91 \pm 1.30 \%$ (note that these scores are averaged over all signal durations); $\left.\quad F_{1,325.99}=29.95, \quad P<0.001\right]$ and age (young: $65.39 \pm 1.27 \%$; old: $55.98 \pm 1.20 \% \quad$ hits; $\quad F_{1,325.99}=29.08$,
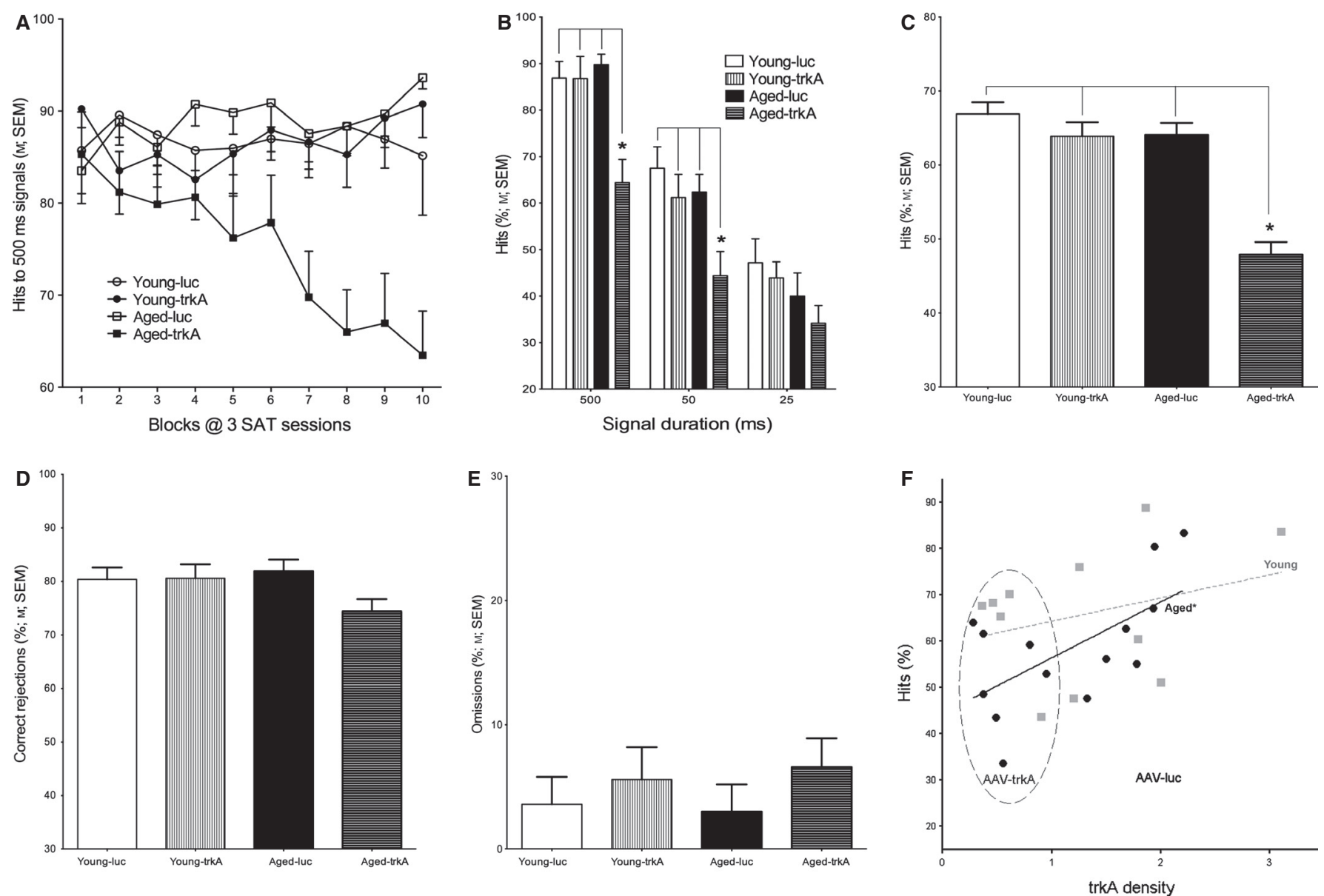

FIG. 4. Effects of nBM/SI trkA knockdown on attentional performance of young and aged animals. (A) Hits to longest signals during the initial approximately 4 weeks post-infusion. This measure began to decline in aged rats during the third week post-infusion. (B) The effects of the vector and age interacted to impair the animals' performance in signal trials (see Results for main statistical findings) but not in non-signal trials (D). Multiple comparisons are based on main effects and a significant interaction between the effects of vector, age and signal duration (LSD: $* P<0.05$, **P<0.001). (C) Post hoc one-way ANOvA of the effects of group confirmed the selective impairment in aged/AAV-trkA rats. (E) Omission remained low $(<8 \%)$ and did not differ between conditions. (F) Correlations between SAT scores and nMB/SI trkA density indicated a significant positive correlation in aged but not young rats (see Results). Data points from animals infused with AAV-trkA are all within the circled area, reflecting a complete separation of trkA levels as a function of vector. Additional analysis also indicated that trkA levels in (young and aged) control animals generally correlated with the animals' hit rate; infusions of AAV-trkA abolished this relationship. 
$P<0.001$ ). Post hoc analyses (Fig. 4B) indicated that aged animals infused with trkA-AAV made fewer hits to 500 -ms $\left(F_{3,36}=9.44\right.$, $P<0.001)$ and 50 -ms signals $\left(F_{3,36}=4.97, P=0.005\right.$; LSD all $P<0.02)$. Because of 'floor effects', hits to shortest signal did not differ significantly between the four groups of animals $\left(F_{3,36}=1.50, P=0.232\right)$. The main conclusion that trkA suppression affected primarily performance of aged rats was also indicated by a post hoc one-way ANOvA over groups that again indicated that aged, trkA-AAV-infused rats exhibited lower hit rates than the other three groups $\left(F_{3,36}=5.69, P=0.003\right.$; LSD all $P<0.007$; Fig. $\left.4 C\right)$. In contrast to these effects on the detection of signals, the relative number of correct rejections (Fig. 4D) and errors of omissions (Fig. 4E; $<8 \%$ across conditions) remained unaffected by infusion of the trkA-targeting or control constructs (all $P>0.10$ ).

\section{Correlation with trkA levels}

The results described above indicate that in aged rats, suppression of trkA receptor levels in corticopetal cholinergic neurons resulted in a decrease in the number of correct responses specifically during signal trials (or hits). We therefore analysed the relationship, for each age group, between levels of nMB/SI trkA, determined using immunohistochemistry and densitometry (see Materials and methods and Fig. 7) and the animals' hit rates averaged over the last three sessions before tissue processing. As illustrated in Fig. 4F, data points depicting AAV-trkA-induced reduction in trkA (left circled cloud) are clearly separated from trkA levels in AAV-luc-infused rats. Hit rates and trkA levels were positively correlated in aged (Pearson's $r=0.62, P=0.02)$ but not young rats $(r=0.296$, $P=0.33)$. Inspection of the scatterplot also indicated that among animals infused with the control vector (young and aged), trkA levels generally correlated with hits $(r=0.56, P=0.049)$. Infusion of AAV-trkA abolished this relationship $(P=0.35)$.

\section{$A A V$-trkA reduces prefrontal $A C h$ release capacity in aged rats}

Brief, second-based release of ACh in medial prefrontal cortex mediates the detection of signals in attentional tasks (Parikh et al.,
2007; Parikh \& Sarter, 2008; Howe et al., 2010; Hasselmo \& Sarter, 2011). As the infusion of trkA-AAV selectively decreased the hit rates in aged animals, we hypothesized that the capacity of prefrontal terminals to release ACh was attenuated. ACh release capacity was assessed by depolarizing cholinergic terminals with $\mathrm{KCl}$ (70 $\mathrm{mm})$, pressure-ejected into the recording regions via coimplanted micropipettes (see Materials and methods; e.g. Parikh \& Sarter, 2006).

Following completion of behavioral experiments, five animals from each group were randomly selected and prepared for electrochemical recordings of evoked ACh release in prefrontal cortex. Choline-sensitive microelectrodes exhibited a sensitivity of $7.11 \pm 0.75 \mathrm{pA} / \mu \mathrm{M}$, an LOD for choline of $375.05 \pm 37.54 \mathrm{~nm}$, and their selectivity of choline over ascorbic acid was $139.73 \pm 20.40$.

As illustrated in Fig. 5, the amplitudes of $\mathrm{KCl}$-evoked $\mathrm{ACh}$ release events were significantly reduced by trkA knockdown $\left(F_{1,16}=30.38, \quad P<0.001\right)$ and age $\left(F_{1,16}=7.67, \quad P=0.014\right)$. Importantly, the effects of these two factors interacted significantly $\left(F_{1,16}=4.52, P=0.049\right)$. Post hoc multiple comparisons (Fig. 5B) indicated significantly attenuated $\mathrm{ACh}$ release in aged animals infused with AAV-trkA when compared with the other three groups (LSD - all $P<0.003$ ). Furthermore, amplitudes did not differ between young and aged animals infused with AAV-Luc $(P=0.66)$. Thus, age interacted with the effects of trkA knockdown to attenuate the capacity of cholinergic terminals to release ACh. Paralleling the significant correlation between trkA levels in the $\mathrm{nMB} / \mathrm{SI}$ region and the relative number of hits of aged rats (above), smaller amplitudes of $\mathrm{KCl}$-evoked ACh release likewise correlated with the hit rate of aged animals $(r=0.86, P=0.002$; young: $P=0.11)$.

\section{AAV-trkA did not affect cholinergic cell counts and size but reduced somatic and cortical ChAT expression}

ChAT is a specific phenotypic marker of cholinergic neurons (Oda \& Nakanishi, 2000). Because NGF-trkA signaling is essential for the maturation of cholinergic neurons (Li et al., 1995; Fagan et al., 1997) as well as influencing ACh synthesis and storage, in part by
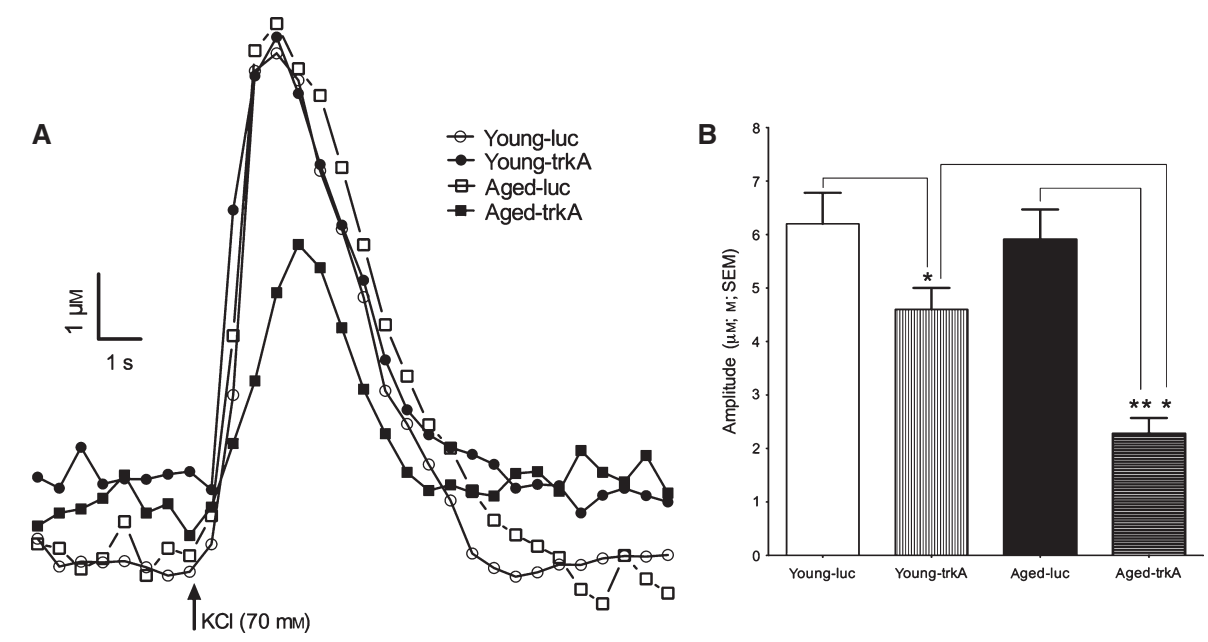

FIG. 5. Depolarization-evoked ACh release in prefrontal cortex. (A) Representative traces of choline signals recorded amperometrically using choline-sensitive microelectrodes placed in the prelimbic regions of the medial prefontal cortex. Release was evoked by pressure ejections of KCl via co-implanted micropipettes. These traces illustrate the attenuated amplitude of evoked ACh release in aged/AAV-trkA rats. (B) A significant interaction between the effects of age and vector (Results) reflect the greater AAV-trkA-induced attenuation of ACh release capacity in aged animals when compared with young animals $(\mathrm{LSD}$ : $* P<0.05$, $* * P<0.001)$. 
upregulating the expression of ChAT (Pongrac \& Rylett, 1998; Oosawa et al., 1999; Szutowicz et al., 2004), we determined the effects of age and trkA knockdown on the integrity of cholinergic neurons in the nMB/SI region. Because in the current study trkA receptor knockdown occurred in adult animals, cholinergic cell loss was not expected to result from infusions of AAV-trkA.

ChAT-immunoreactive neurons in the $\mathrm{nMB} / \mathrm{SI}$ region are illustrated in Fig. 6A. The total numbers of ChAT-positive neurons along the rostral-caudal axis of the nBM/SI region of animals ranged from $8429 \pm 1362$ in young/AAV-luc rats to $7326 \pm 1468$ in aged/AAV-trkA rats and did not differ among the groups (main effects of vector, age and interaction; all $P>0.47$ ). Morphometric analyses of ChAT-positive cells (see Materials and methods) indicated that the mean cross-sectional area of BF cholinergic cells was significantly smaller in aged than in young animals $\left(F_{1,12}=27.03\right.$,
$P<0.001$; Fig. 6B). However, there was no effect of the vector and the two factors did not interact (both $P>0.14$ ).

In contrast, levels of ChAT expression in the region of the nMB/ SI was affected by age $\left(F_{1,12}=135.44, P<0.001\right)$ and vector $\left(F_{1,12}=5.77, P=0.03\right)$, and the two factors interacted significantly $\left(F_{1,12}=5.10, P=0.04\right.$; Fig. $\left.6 \mathrm{C}\right)$. Multiple comparisons indicated that ChAT levels did not differ between AAV-luc- and AAV-trkAinfused young animals but were generally lower in aged rats, and lower in aged/AAV-trkA than in aged rats that received infusions of the control vector (Fig. 6C).

Although, as expected, trkA knockdown in adult rats did not affect the integrity of cholinergic neurons in the $\mathrm{BF}$, in vitro studies indicated that synaptic mechanisms, including expression of the high-affinity choline transporter (Madziar et al., 2008), vesicular storage of ACh and release mechanisms (Oosawa et al., 1999), are
A
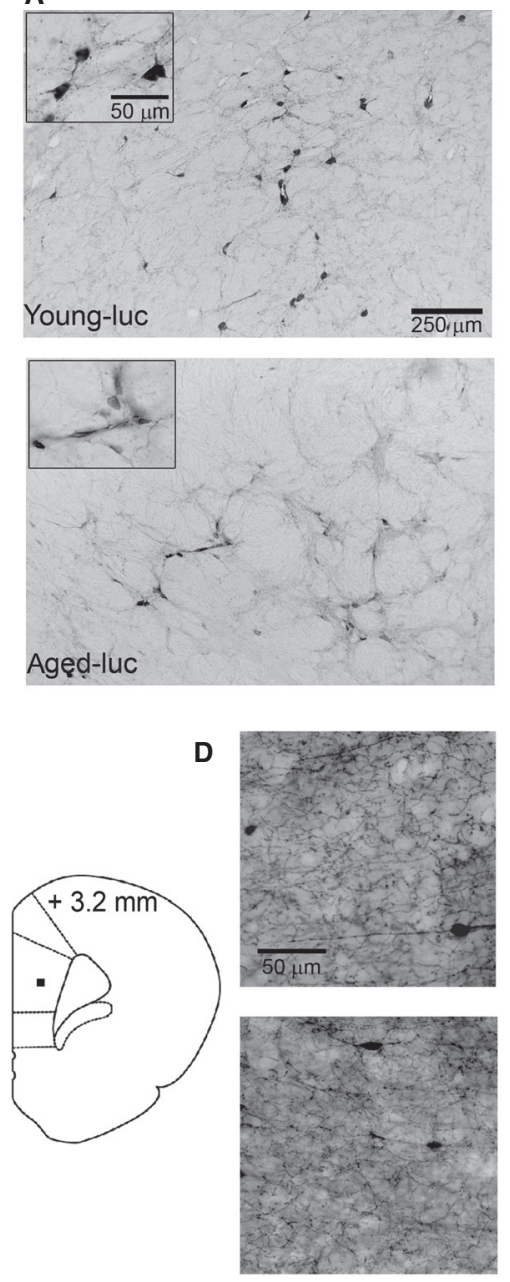

Young
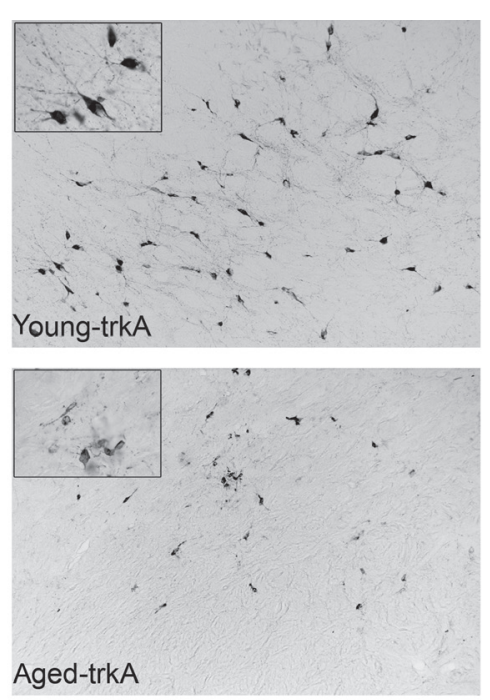

B
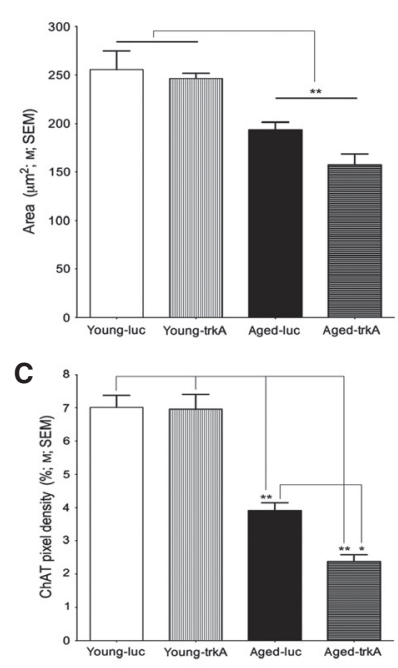
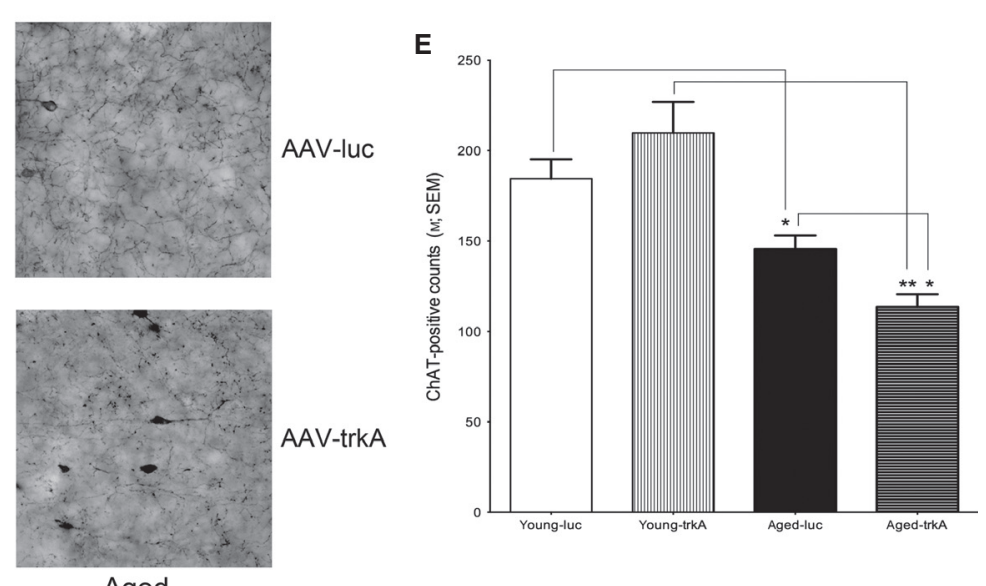

Aged

FIG. 6. Cholinergic neurons in the nMB/SI and cortical cholinergic processes in young and aged animals after AAV-mediated trkA knockdown. Sections were processed for ChAT immunohistochemistry. (A) Representative images from coronal sections depicting ChAT-immunopositive neurons in the nBM/SI region. The inset shows examples of cholinergic neurons at a higher magnification. As described in the Results, neither age nor vector affected cholinergic cell counts. (B) Cholinergic cell area was reduced in aged animals as compared with young animals; however, this measure was not affected by vector and the effects of age and vector did not interact (main effect of age: $* *, P<0.001$ ). (C) ChAT levels expressed as percentage ChAT-positive pixels in the analysed area (see Materials and methods). There was no discernible difference in ChAT levels between young AAV-luc and AAV-trkA animals. Multiple comparisons were based on significant effects of and interaction between age and vector. ChAT levels declined with age and were lowest in aged/AAV-trkA rats. (D, E) Paralleling ChAT levels in the nMB/SI region, the density of ChAT-positive puncta in the prefrontal cortex was significantly lower in aged animals in general, and lower in aged/AAV-trkA rats when compared with aged control (aged/AAV-luc) and young/AAV-trkA rats. The counting area is symbolized in the coronal schematic on the left. Note that darkstained cholinergic, bipolar interneurons were not included into cortical counts (multiple comparisons for C and E; LSD: ${ }^{*} P<0.05,{ }^{* *} P<0.01$ ). 
regulated by NGF-trkA signaling. Therefore, we also examined the integrity of cholinergic processes in the cortex, specifically in the prelimbic region in which ACh release capacity was measured (above). Importantly, quantification of ChAT-positive processes excluded cortical ChAT-positive bipolar interneurons that are largely located in layers 2-3 (von Engelhardt et al., 2007) and may not depend on NGF signaling (Heckers et al., 1994). Repeated-measures mixed-factor ANOva indicated a main effect of age $\left(F_{1,11}=41.19\right.$, $P=0.001)$, no main effect of vector $\left(F_{1,11}=0.11, P=0.75\right)$, but a significant interaction between both factors $\left(F_{1,11}=7.37, P=0.02\right)$. Multiple comparisons (see Fig. 6D and E) indicated that this interaction reflected a lower density of cholinergic processes in aged/AAVtrkA rats when compared with all other three groups. Furthermore, immunoblot analysis of prefrontal ChAT expression (see Supporting Information) supports this view.

\section{proNGF/NGF and p75/trkA balances - effects of age and trkA silencing}

An imbalance between the stoichiometry of trkA and p75 receptors is hypothesized to trigger cholinergic decline by producing a shift from pro-survival to apoptotic signaling during normal and pathological aging (Costantini et al., 2005, 2006; Mufson et al., 2007). Moreover, an age-related increase in levels of proNGF, a precursor form of NGF that binds preferentially to 75 receptors, also contributes to the activation of apoptotic pathways (Coulson et al., 2009). Therefore, we determined whether trkA knockdown evokes changes in the levels of proNGF and NGF and p75 receptor densities. Because NGF is synthesized in the cortex and retrogradely transported to nBM/SI cholinergic neurons, NGF and proNGF levels were assessed in prefrontal cortex. Alterations in p75 and trkA receptor expression were evaluated in the cortex as well as nMB/SI cholinergic neurons.

ELISA indicated neither main effects of age or vector nor an interaction between the effects of these two factors on prefrontal levels of NGF (all $P>0.63$ ). Immunoblot analyses indicated that, consistent with the results of prior studies (Al-Shawi et al., 2007; Terry et al., 2011), aging increases cortical levels of proNGF expression $\left(F_{1,9}=68.04, P<0.001\right)$. Furthermore, higher levels of proNGF were observed following the infusion of AAV-trkA $\left(F_{1,9}=9.48\right.$, $P=0.01$; Fig. 7A). However, there was no interaction between these two factors $(P=0.94)$.

Cortical p75 receptor expression was elevated in aged animals $\left(F_{1,9}=63.44, P<0.001\right.$; Fig. 7 B $)$ but not by vector $\left(F_{1,9}=3.46\right.$, $P=0.09)$ and the two factors did not interact $\left(F_{1,9}=0.17\right.$, $P=0.69)$. As expected, infusions of AAV-trkA lowered trkA levels in the cortex $\left(F_{1,9}=19.51, P<0.001\right.$; Fig. $\left.7 C\right)$ as well as the nMBI/SI $\left(F_{1,14}=29.42, P<0.001\right.$; Fig. 7D and E). However, neither cortical nor basal forebrain trkA levels were affected by age (both $P>0.75$ ) nor did the two factors interact (both $P>0.78$ ). Finally, p75 levels in the nMB/SI remained unaffected by age and vector (all $P>0.27$; Fig. $7 \mathrm{~F}$ and $\mathrm{G}$ ).

\section{Discussion}

The results from our experiments support the following conclusions. (i) Our vector-based RNAi approach chronically suppressed trkA receptor in the corticopetal cholinergic projection system of adult rats. Our strategy produced approximately $70 \%$ knockdown of these receptors in the $\mathrm{nBM} / \mathrm{SI}$ region and a approximately $55 \%$ reduction in cortical trkA levels. (ii) Suppression of trkA receptor expression in aged but not young-adult rats (see Results for definition and characterization of aged rats) resulted in a selective decrease in their attentional performance. This impairment manifested at around 3 weeks post-infusion, consistent with the time course of trkA suppression observed in our initial characterization of vector efficacy. In animals with intact trkA levels, attentional performance, specifically hit rates, correlated significantly with trkA levels. In aged rats, lower attentional performance was correlated with lower trkA levels expressed in nMB/SI cholinergic neurons. (iii) After completion of the behavioral experiments the capacity of cholinergic terminals in the cortex to release ACh in vivo was attenuated by trkA suppression in general and was significantly lower in aged rats infused with the vector than in their young counterparts. ACh release amplitudes recorded in aged rats correlated significantly with their attentional performance. (iv) Morphometric analyses indicated that neither age nor the vector affected the number of cholinergic cells in the $\mathrm{nMB} / \mathrm{SI}$. The size of cholinergic neurons was reduced in aged rats but these measures were not affected by trkA suppression. In contrast, ChAT levels in the nBM/SI region and the density of ChAT-positive processes in the cortex was lower in aged rats and further reduced as a result of trkA suppression, yielding lower somatic and cortical ChAT-positive densities in aged/trkA-AAV rats than in aged control and young/trkA-AAV rats. (v) A final series of experiments indicated that aging and trkA suppression increased cortical proNGF levels and that the former also increased cortical p75 receptor densities in the cortex.

Although several experiments have demonstrated age-related changes in cholinergic markers in the rodent brain, our own prior studies measuring, in vivo, cholinergic neurotransmission in awake and performing rats show that the capacity of cholinergic systems to support cognitive performance is not robustly impaired in aged rats (reviewed by Sarter \& Bruno, 2004). This statement excludes the more general and unspecific decline in behavioral performance often seen in aged but premorbid rats. Because age per se does not appear to be a useful variable when using rats to study cholinergic functions (Parikh \& Sarter, 2010), the modeling of age-related cholinergic vulnerabilities requires additional experimental manipulations. For example, we previously demonstrated that limited cortical cholinergic deafferentation reveals age-related decline in cholinergic and associated cognitive abilities (Burk et al., 2002). However, as postmortem analyses of brains from patients with mild cognitive impairment or early stage AD indicated stable cholinergic cell counts but robust loss of trkA receptor density (Mufson et al., 2000; Chu et al., 2001; Counts et al., 2004; Saragovi, 2005; Ginsberg et al., 2006), suppression of basal forebrain trkA receptor expression in aged rats may represent a more valid model.

We have employed a vector-based RNAi approach to exclude the consequences of disruption of NGF-trkA signaling during development on the maturation, cell survival, axon guidance and integrity of cholinergic neurons (Fagan et al., 1997; Chao, 2003; SanchezOrtiz et al., 2012). Moreover, this approach allowed us to test the role of trkA signaling specifically in the $\mathrm{nMB} / \mathrm{SI}$ cholinergic projection system in the adult and aged brain, and to exclude behavioral confounds resulting from trkA reduction elsewhere in the nervous system (Holtzman et al., 1995; Luther \& Birren, 2009). Additionally, the use of adult trkA knockdown accurately models the decrease in BF trkA but not p75 levels, as well as the preservation of nMBI/SI cholinergic neurons observed in patients prior to or in early-stage AD (e.g. Counts et al., 2004).

Our results are consistent with the recent finding that mice with a forebrain-specific deletion of trkA gene do not exhibit alterations in the number of BF cholinergic neurons and cell size (Sanchez-Ortiz et al., 2012). However, in contrast to the findings by these authors, we did not observe a reduction in BF ChAT expression in trkA 
A
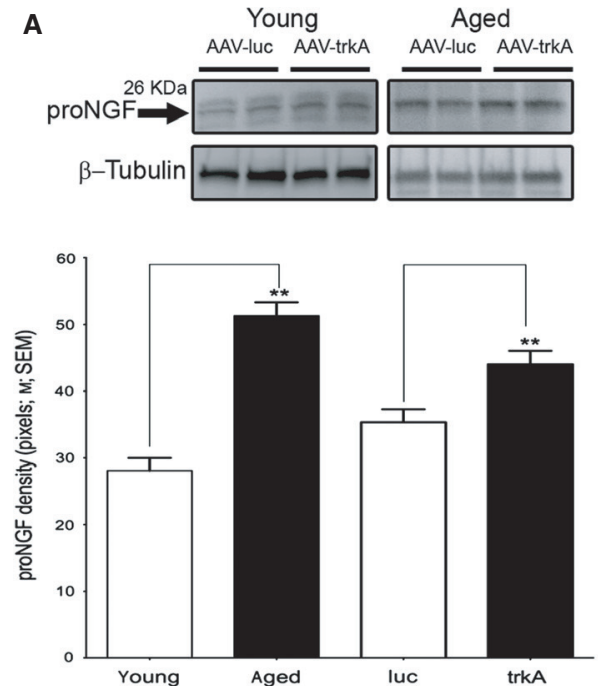

D

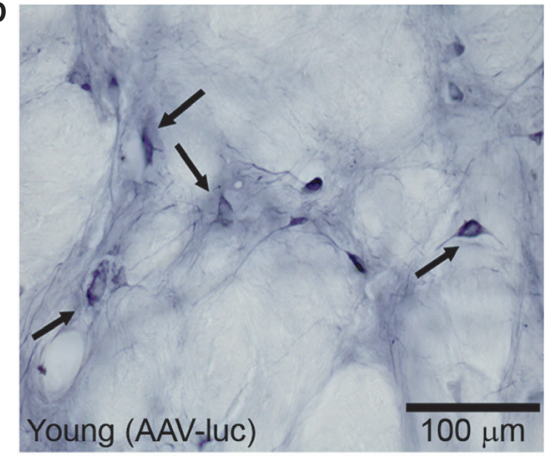

$\mathbf{F}$

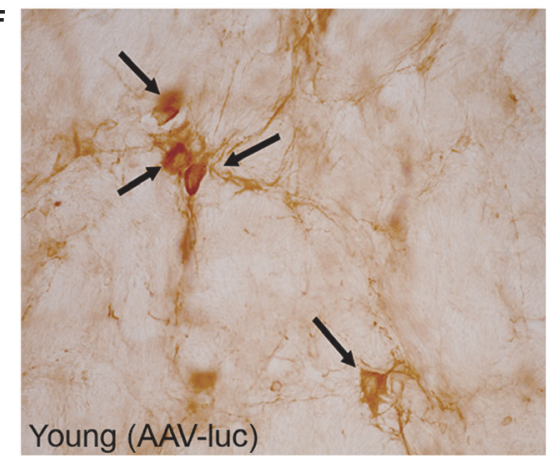

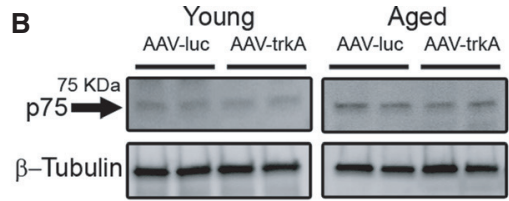
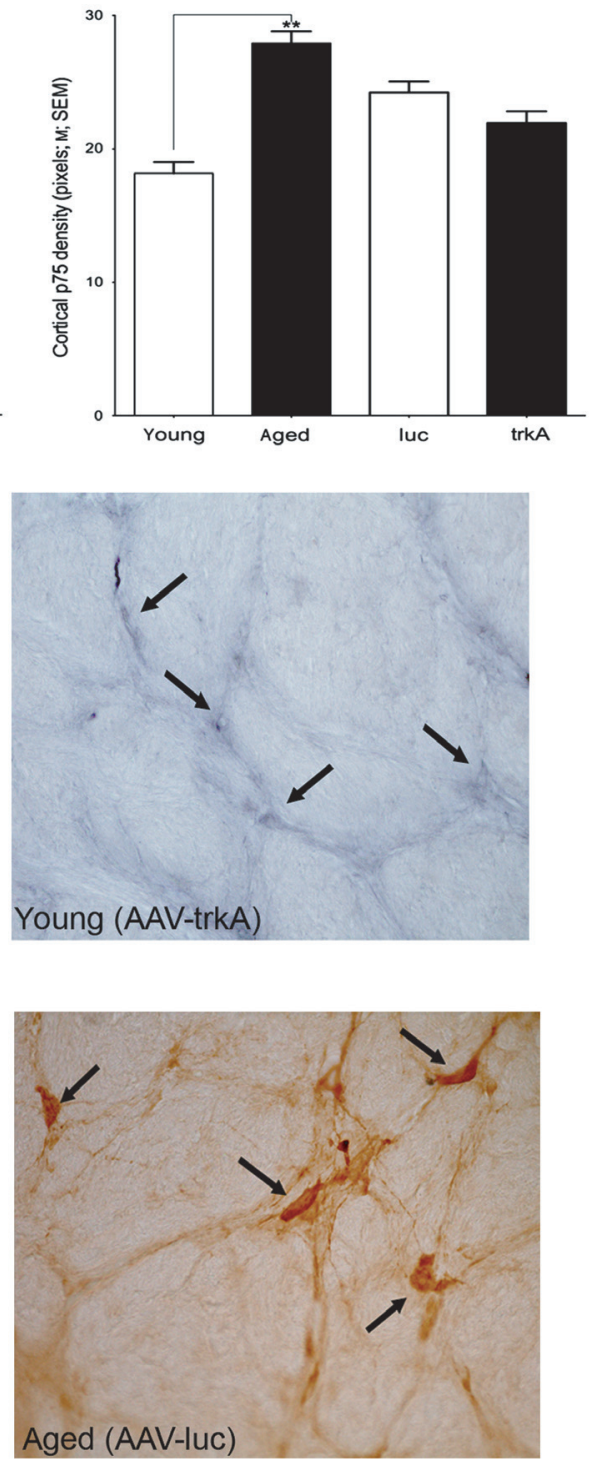

C
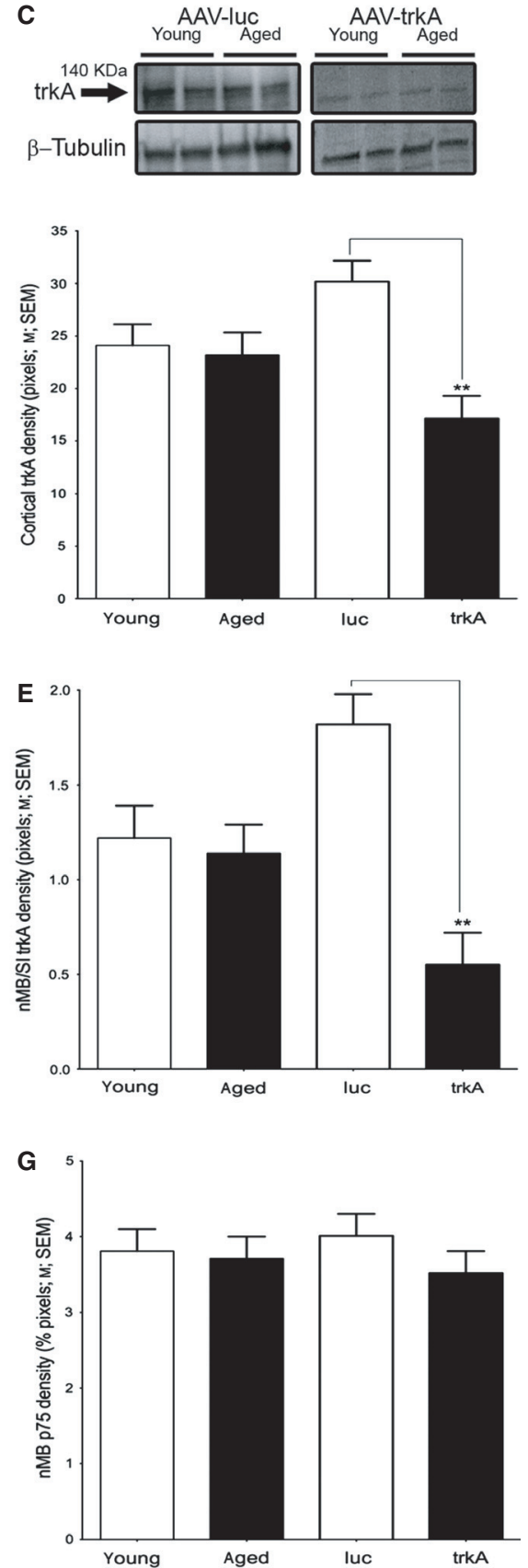

FIG. 7. Effects of age and trkA knockdown on cortical NGF/proNGF levels and cortical and basal forebrain NGF receptor densities. NGF levels were estimated in cortical homogenates using ELISA. Neither age nor infusion of the vector affected cortical NGF levels (not shown). (A) In contrast, proNGF levels were higher in aged rats and in rats infused with AAV-trkA vector. Representative immunoblots of cortical proNGF from duplicate samples are shown at the top. A proNGF immunoreactive band (26 kDa) was detected using a rabbit monoclonal anti-proNGF antibody. Bar graphs (bottom) show proNGF densities normalized to $\beta$-tubulin (LSD: **, $P \leq 0.01$ ). (B) Cortical p75 receptor density was increased in aged animals but remained unaffected by infusion of the vector. Blots depict a $75-\mathrm{kDa}$ band corresponding to p75 receptor. (C) Cortical trkA receptor density was unaffected by age but, as expected, decreased as a result of infusion of the AAV-trkA vector. (D, E) trkA receptor-immunopositive neurons in the nMB/SI region of young rats infused with either the control or the AAV-trkA vector (left, right in D). Similar to cortical trkA levels, nMBI/SI levels were unaffected by age but robustly suppressed by the AAV-trkA vector (E). (F, G) p75 receptor expression in the nMB/SI region was affected neither by age nor by infusion of the AAV-trkA vector (see Discussion for the contrasting age-related increase in cortical p75 levels; see Results for ANovas; post hoc comparisons for B, C, E and G: **P<0.001 young vs. aged or luc vs. trkA).

knockdown young animals. As the level of ligand but not trkA density is considered to be the rate-limiting factor for the maintenance of cholinergic neurons (Fagan et al., 1997), it is possible that, in our study, NGF or other neurotrophins preserved the integrity of cortical cholinergic inputs. The present evidence indicating the absence of a correlation between BF trkA densities and SAT scores and the reduction in depolarization-evoked cortical ACh release in young animals further support this hypothesis. Therefore, NGF-trkA signaling may be required but not essential for the survival and growth of cortically projecting forebrain cholinergic neurons and for the associated preservation of attentional capacities in adult rats. Nevertheless, this perspective does not rule out an essential role of 
trkA signaling in the maintenance of cholinergic function during early development (Fagan et al., 1997; Sarter \& Bruno, 2004; Sanchez-Ortiz et al., 2012).

However, disruption of trkA signaling in the aged brain impacted cholinergic function. It reduced cortical ChAT-positive processes and ACh release capacity. Loss of ChAT immunoreactive fibers and axonal varicosities indicate cholinergic deafferentation (Garofalo et al., 1992). As choline uptake via high-affinity choline transporters, and not the acetylation of choline by ChAT, is the rate-limiting step for ACh synthesis (Ferguson \& Blakely, 2004; Sarter \& Parikh, 2005), our findings further indicate that reduced cholinergic transmission in trkA knockdown aged rats was a consequence of a reduced density of presynaptic cholinergic terminals.

Increased levels of cortical proNGF (see also Peng et al., 2004) coincided with increased p75 receptors in aged rats. Both of these effects of age mediate primarily apoptotic mechanisms, with the former involving sortilin receptor-mediated signaling (Chao, 2003; Costantini et al., 2006; Volosin et al., 2006; Al-Shawi et al., 2007, 2008; Jansen et al., 2007; Clewes et al., 2008; Sobottka et al., 2008; Fortress et al., 2011). Although our analyses of proNGF, NGF, p75 and trkA receptor levels did not reveal interactions between the effects of age and trkA suppression, the combination of these effects of aging and trkA suppression may be sufficient to explain the greater vulnerability of the aged cholinergic system to trkA suppression. As aging alone increases proNGF and p75 levels, the interactions between these effects and the experimental attenuation of trkA receptor levels shifts the balance of NGF-induced signaling strongly toward p75-mediated suppression of the cholinergic phenotype (see also Szutowicz et al., 2004; Terry et al., 2011). Conversely, the detrimental consequences of trkA receptor expression in young-adult brains remained limited presumably because they did not interact with elevated p75 levels and high proNGF levels.

In this context, the contrast between the age-related increase in p75 levels in the cortex and the absence of such an effect of age on p75 immunoreactivity in the nMB/SI requires comment. Age-related increases in p75 levels in the cortex of rodents were previously reported (Costantini et al., 2005; Terry et al., 2011). It may be speculated that terminal p75 receptor densities, similar to sortilin receptor levels (Al-Shawi et al., 2007), increase in aging as a function of increased cortical proNGF levels (Fig. 7A). Consistent with this speculation, infusions of proNGF were demonstrated to increase telencephalic p75 levels in rats (Fortress et al., 2011). In conjunction with lower, vector-induced trkA expression, these changes promote suppression of the cholinergic phenotype that manifests initially in terms of ACh release capacity (Fig. 5) and the density of cortical cholinergic inputs (Fig. 6). In the long term, such trophic alterations and the resulting decline in cholinergic neurotransmission may activate additional neurotoxic mechanisms, including amyloid beta peptide, leading to cholinergic and non-cholinergic cell loss (Costantini et al., 2005; Coulson et al., 2009).

Abnormal trophic regulation represents a major cause of the decline in forebrain cholinergic systems and associated cognitive capacities (e.g. Counts \& Mufson, 2005; Costantini et al., 2006). Our results indicate that suppression of trkA receptor expression in aged rats is a model of such age-related cholinergic vulnerability, including the underlying shift toward p75 signaling that contributes to attenuated cholinergic innervation of the cortex. This model reproduces key aspects of the cholinergic system in patients with early-stage or forthcoming $\mathrm{AD}$, including stable cholinergic cell numbers but decreased cortical cholinergic processes, lower trkA but stable p75 levels, stable cortical NGF but elevated proNGF lev- els and, importantly, a cognitive phenotype that closely reflects the functional state of the cholinergic system. Thus, this model will be useful to further research on the cellular, neuronal and psychobiological mechanisms of pathological aging of the cholinergic system. Finally, this study provides novel evidence suggesting that reduced expression of forebrain trkA receptors is not sufficient to trigger cholinergic dysfunction. Rather, aging interacts with disrupted trkA signaling, to escalate the vulnerability of cortical cholinergic inputs and the manifestation of age-related cognitive impairments.

\section{Supporting Information}

Additional supporting information can be found in the online version of this article:

Data S1. Methods.

Fig. S1. Forebrain trkB receptor expression was not affected by trkA shRNA.

Fig. S2. Effects of age and vector manipulation on cortical ChAT expression.

\section{Acknowledgements}

This work was supported by grants from the National Institute on Aging (AG029592, V.P. \& M.S.), the Rosalinde and Arthur Gilbert Foundation/ American Federation for Aging Research (V.P.), and the National Institute of Neurological Disorders and Stroke (NS38698, D.L.T.). Thanks to Ms Carcha Bernard and Brittany Tracy (Temple University) for assistance with cell culture and immunocytochemistry, and to Melissa Tippens (University of Michigan) for assistance with initial construction of the AAV-SIBR vector. We also thank Drs Diana S. Woodruff-Pak and John Kennard (Temple University) for assisting us with unbiased stereology. The University of Michigan has a patent on miR-155-based RNAi technology. D.L.T. is a potential recipient of royalties paid to the University of Michigan for licensed use.

\section{Abbreviations}

AA, ascorbic acid; AAV, adeno-associated viral; ACh, acetylcholine; AD, Alzheimer's disease; BF, basal forebrain; DA, dopamine; DAB, 3-3'-diaminobenzidine; GFP, green fluorescent protein; HRP, horseradish peroxidase; ITI, intertrial interval; ITRs, inverted terminal repeats; LOD, limit of detection; LSD, least significant difference; luc, luciferase; NGF, nerve growth factor; nMB/SI, nucleus basalis of Meynert/substantia innominata; polyA, polyadenylation; RNAi, RNA interference; SAT, sustained attention task shRNAs, short hairpin RNAs; SIBR, synthetic BIC-derived RNA; TBS, Trisbuffered saline; trkA, tropomyosin-related kinase A; Ubc, human ubiquitin $\mathrm{C}$.

\section{References}

Al-Shawi, R., Hafner, A., Chun, S., Raza, S., Crutcher, K., Thrasivoulou, C., Simons, P. \& Cowen, T. (2007) ProNGF, sortilin, and age-related neurodegeneration. Ann. NY Acad. Sci., 1119, 208-215.

Al-Shawi, R., Hafner, A., Olson, J., Chun, S., Raza, S., Thrasivoulou, C. Lovestone, S., Killick, R., Simons, P. \& Cowen, T. (2008) Neurotoxic and neurotrophic roles of proNGF and the receptor sortilin in the adult and ageing nervous system. Eur. J. Neurosci., 27, 2103-2114.

Apparsundaram, S., Martinez, V., Parikh, V., Kozak, R. \& Sarter, M. (2005) Increased capacity and density of choline transporters situated in synaptic membranes of the right medial prefrontal cortex of attentional task-performing rats. J. Neurosci., 25, 3851-3856.

Burk, J.A., Herzog, C.D., Porter, M.C. \& Sarter, M. (2002) Interactions between aging and cortical cholinergic deafferentation on attention. Neurobiol. Aging, 23, 467-477.

Ceni, C., Kommaddi, R.P., Thomas, R., Vereker, E., Liu, X., McPherson, P.S., Ritter, B. \& Barker, P.A. (2010) The p75NTR intracellular domain generated by neurotrophin-induced receptor cleavage potentiates Trk signaling. J. Cell Sci., 123, 2299-2307.

Chao, M.V. (2003) Neurotrophins and their receptors: a convergence point for many signaling pathways. Nat. Rev. Neurosci., 4, 299-309. 
Chu, Y., Cochran, E.J., Bennett, D.A., Mufson, E.J. \& Kordower, J.H. (2001) Down-regulation of trkA mRNA within nucleus basalis neurons in individuals with mild cognitive impairment and Alzheimer's disease. J. Comp. Neurol., 437, 296-307.

Chung, K.H., Hart, C.C., Al-Bassam, S., Avery, A., Taylor, J., Patel, P.D., Vojtek, A.B. \& Turner, D.L. (2006) Polycistronic RNA polymerase II expression vectors for RNA interference based on BIC/miR-155. Nucleic Acids Res., 34, e53.

Clewes, O., Fahey, M.S., Tyler, S.J., Watson, J.J., Seok, H., Catania, C., Cho, K., Dawbarn, D. \& Allen, S.J. (2008) Human ProNGF: biological effects and binding profiles at TrkA, P75NTR and sortilin. J. Neurochem., 107, 1124-1135.

Costantini, C., Weindruch, R., Della Valle, G. \& Puglielli, L. (2005) A TrkA-to-p75NTR molecular switch activates amyloid beta-peptide generation during aging. Biochem. J., 391, 59-67.

Costantini, C., Scrable, H. \& Puglielli, L. (2006) An aging pathway controls the TrkA to p75NTR receptor switch and amyloid beta-peptide generation. EMBO J., 25, 1997-2006.

Coulson, E.J., May, L.M., Sykes, A.M. \& Hamlin, A.S. (2009) The role of the p75 neurotrophin receptor in cholinergic dysfunction in Alzheimer's disease. Neuroscientist, 15, 317-323.

Counts, S.E. \& Mufson, E.J. (2005) The role of nerve growth factor receptors in cholinergic basal forebrain degeneration in prodromal Alzheimer disease. J. Neuropathol. Exp. Neurol., 64, 263-272.

Counts, S.E., Nadeem, M., Wuu, J., Ginsberg, S.D., Saragovi, H.U. \& Mufson, E.J. (2004) Reduction of cortical TrkA but not p75(NTR) protein in early-stage Alzheimer's disease. Ann. Neurol., 56, 520-531.

Das, K.P., Freudenrich, T.M. \& Mundy, W.R. (2004) Assessment of PC12 cell differentiation and neurite growth: a comparison of morphological and neurochemical measures. Neurotoxicol. Teratol., 26, 397-406.

Demeter, E., Sarter, M. \& Lustig, C. (2008) Rats and humans paying attention: cross-species task development for translational research. Neuropsychology, 22, 787-799.

Dickson, H.M., Zurawski, J., Zhang, H., Turner, D.L. \& Vojtek, A.B. (2010) POSH is an intracellular signal transducer for the axon outgrowth inhibitor Nogo66. J. Neurosci., 30, 13319-13325.

von Engelhardt, J., Eliava, M., Meyer, A.H., Rozov, A. \& Monyer, H. (2007) Functional characterization of intrinsic cholinergic interneurons in the cortex. J. Neurosci., 27, 5633-5642.

Everitt, B.J. \& Robbins, T.W. (1997) Central cholinergic systems and cognition. Ann. Rev. Psychol., 48, 649-684.

Fagan, A.M., Garber, M., Barbacid, M., Silos-Santiago, I. \& Holtzman, D.M. (1997) A role for TrkA during maturation of striatal and basal forebrain cholinergic neurons in vivo. J. Neurosci., 17, 7644-7654.

Ferguson, S.M. \& Blakely, R.D. (2004) The choline transporter resurfaces: new roles for synaptic vesicles? Mol. Interv., 4, 22-37.

Fortress, A.M., Buhusi, M., Helke, K.L. \& Granholm, A.C. (2011) Cholinergic degeneration and alterations in the TrkA and p75NTR balance as a result of Pro-NGF injection into aged rats. J. Aging Res., 2011, 460543.

Garofalo, L., Ribeiro-da-Silva, A. \& Cuello, A.C. (1992) Nerve growth factor-induced synaptogenesis and hypertrophy of cortical cholinergic terminals. Proc. Natl. Acad. Sci. USA, 89, 2639-2643.

Ginsberg, S.D., Che, S., Wuu, J., Counts, S.E. \& Mufson, E.J. (2006) Down regulation of trk but not p75NTR gene expression in single cholinergic basal forebrain neurons mark the progression of Alzheimer's disease. J. Neurochem., 97, 475-487.

Greenwald, A.G., Gonzalez, R., Harris, R.J. \& Guthrie, D. (1996) Effect sizes and $P$ values: what should be reported and what should be replicated? Psychophysiology, 33, 175-183.

Hartikka, J. \& Hefti, F. (1988) Comparison of nerve growth factor's effects on development of septum, striatum, and nucleus basalis cholinergic neurons in vitro. J. Neurosci. Res., 21, 352-364.

Hasselmo, M.E. \& Sarter, M. (2011) Modes and models of forebrain cholinergic neuromodulation of cognition. Neuropsychopharmacol., 36, 52-73.

Heckers, S., Ohtake, T., Wiley, R.G., Lappi, D.A., Geula, C. \& Mesulam, M.M. (1994) Complete and selective cholinergic denervation of rat neocortex and hippocampus but not amygdala by an immunotoxin against the p75 NGF receptor. J. Neurosci., 14, 1271-1289.

Holtzman, D.M., Kilbridge, J., Li, Y., Cunningham, E.T., Lenn, N.J., Clary, D.O., Reichardt, L.F. \& Mobley, W.C. (1995) TrkA expression in the CNS: evidence for the existence of several novel NGF-responsive CNS neurons. J. Neurosci., 15, 1567-1576.

Hommel, J.D., Sears, R.M., Georgescu, D., Simmons, D.L. \& DiLeone, R.J. (2003) Local gene knockdown in the brain using viral-mediated RNA interference. Nat. Med., 9, 1539-1544.
Howe, W.M., Ji, J., Parikh, V., Williams, S., Mocaër, E., Trocmé-Thibierge, C. \& Sarter, M. (2010) Enhancement of attentional performance by selective stimulation of alpha4beta2* nAChRs: underlying cholinergic mechanisms. Neuropsychopharmacol., 35, 1391-1401.

Huang, E.J. \& Reichardt, L.F. (2003) Trk receptors: roles in neuronal signal transduction. Ann. Rev. Biochem., 72, 609-642.

Jansen, P., Giehl, K., Nyengaard, J.R., Teng, K., Lioubinski, O., Sjoegaard, S.S., Breiderhoff, T., Gotthardt, M., Lin, F., Eilers, A., Petersen, C.M., Lewin, G.R., Hempstead, B.L., Willnow, T.E. \& Nykjaer, A. (2007) Roles for the pro-neurotrophin receptor sortilin in neuronal development, aging and brain injury. Nat. Neurosci., 10, 1449-1457.

Kaplan, D.R. \& Miller, F.D. (1997) Signal transduction by the neurotrophin receptors. Curr. Opin. Cell Biol., 9, 213-221.

Koornneef, A., van Logtenstein, R., Timmermans, E., Pisas, L., Blits, B., Abad, X., Fortes, P., Petry, H., Konstantinova, P. \& Ritsema, T. (2011) AAV-mediated in vivo knockdown of luciferase using combinatorial RNAi and U1i. Gene Ther., 18, 929-935.

Kozak, R., Martinez, V., Young, D., Brown, H., Bruno, J.P. \& Sarter, M. (2007) Toward a neuro-cognitive animal model of the cognitive symptoms of schizophrenia: disruption of cortical cholinergic neurotransmission following repeated amphetamine exposure in attentional task-performing, but not non-performing, rats. Neuropsychopharmacol., 32, 2074-2086.

Li, Y., Holtzman, D.M., Kromer, L.F., Kaplan, D.R., Chua-Couzens, J., Clary, D.O., Knüsel, B. \& Mobley, W.C. (1995) Regulation of TrkA and ChAT expression in developing rat basal forebrain: evidence that both exogenous and endogenous NGF regulate differentiation of cholinergic neurons. J. Neurosci., 15, 2888-2905.

Luck, S.J., Ford, J.M., Sarter, M. \& Lustig, C. (2012) CNTRICS final biomarker selection: control of attention. Schizophr. Bull., 38, 53-61.

Luther, J.A. \& Birren, S.J. (2009) p75 and TrkA signaling regulates sympathetic neuronal firing patterns via differential modulation of voltage-gated currents. J. Neurosci., 29, 5411-5424.

Madziar, B., Shah, S., Brock, M., Burke, R., Lopez-Coviella, I., Nickel, A.C., Cakal, E.B., Blusztajn, J.K. \& Berse, B. (2008) Nerve growth factor regulates the expression of the cholinergic locus and the high-affinity choline transporter via the Akt/PKB signaling pathway. J. Neurochem., 107, 1284-1293.

McGaughy, J., Kaiser, T. \& Sarter, M. (1996) Behavioral vigilance following infusions of 192 IgG-saporin into the basal forebrain: selectivity of the behavioral impairment and relation to cortical AChE-positive fiber density. Behav. Neurosci., 110, 247-265.

Mesulam, M. (2004) The cholinergic lesion of Alzheimer's disease: pivotal factor or side show? Learn. Memory, 11, 43-49.

Mesulam, M., Shaw, P., Mash, D. \& Weintraub, S. (2004) Cholinergic nucleus basalis tauopathy emerges early in the aging-MCI-AD continuum. Ann. Neurol., 55, 815-828.

Mobley, W.C., Rutkowski, J.L., Tennekoon, G.I., Gemski, J., Buchanan, K. \& Johnston, M.V. (1986) Nerve growth factor increases choline acetyltransferase activity in developing basal forebrain neurons. Brain Res., 387, 53-62.

Mufson, E.J., Conner, J.M. \& Kordower, J.H. (1995) Nerve growth factor in Alzheimer's disease: defective retrograde transport to nucleus basalis. Neuroreport, 6, 1063-1066.

Mufson, E.J., Ma, S.Y., Cochran, E.J., Bennett, D.A., Beckett, L.A., Jaffar, S., Saragovi, H.U. \& Kordower, J.H. (2000) Loss of nucleus basalis neurons containing trkA immunoreactivity in individuals with mild cognitive impairment and early Alzheimer's disease. J. Comp. Neurol., 427, 19-30.

Mufson, E.J., Counts, S.E., Fahnestock, M. \& Ginsberg, S.D. (2007) Cholinotrophic molecular substrates of mild cognitive impairment in the elderly. Curr. Alzheimer Res., 4, 340-350.

Nistiar, F., Racz, O., Lukacinova, A., Hubkova, B., Novakova, J., Lovasova, E. \& Sedlakova, E. (2012) Age dependency on some physiological and biochemical parameters of male Wistar rats in controlled environment. $J$. Environ. Sci. Health A. Tox. Hazard. Subst. Environ. Eng., 47, 12241233.

Oda, Y. \& Nakanishi, I. (2000) The distribution of cholinergic neurons in the human central nervous system. Histol. Histopathol., 15, 825-834.

Oosawa, H., Fujii, T. \& Kawashima, K. (1999) Nerve growth factor increases the synthesis and release of acetylcholine and the expression of vesicular acetylcholine transporter in primary cultured rat embryonic septal cells. J. Neurosci. Res., 57, 381-387.

Parikh, V. \& Sarter, M. (2006) Cortical choline transporter function measured in vivo using choline-sensitive microelectrodes: clearance of endogenous and exogenous choline and effects of removal of cholinergic terminals. J. Neurochem., 97, 488-503. 
Parikh, V. \& Sarter, M. (2008) Cholinergic mediation of attention: contributions of phasic and tonic increases in prefrontal cholinergic activity. Ann. NY Acad. Sci., 1129, 225-235.

Parikh, V. \& Sarter, M. (2010). Cognitive decline in laboratory animals: models, measures, and validity. In Koob, G., Thompson, R.F. \& LeMoal, M. (Eds), Encyclopedia of Behavioral Neuroscience, Vol. 1. Elsevier, Amsterdam, pp. 294-301.

Parikh, V., Kozak, R., Martinez, V. \& Sarter, M. (2007) Prefrontal acetylcholine release controls cue detection on multiple timescales. Neuron, 56, 141-154.

Parikh, V., Man, K., Decker, M.W. \& Sarter, M. (2008) Glutamatergic contributions to nicotinic acetylcholine receptor agonist-evoked cholinergic transients in the prefrontal cortex. J. Neurosci., 28, 3769-3780.

Parikh, V., Ji, J., Decker, M.W. \& Sarter, M. (2010) Prefrontal beta2 subunitcontaining and alpha7 nicotinic acetylcholine receptors differentially control glutamatergic and cholinergic signaling. J. Neurosci., 30, 3518-3530.

Paxinos, G. \& Watson, C. (2007) The Rat Brain in Stereotaxic Coordinates, Hard Cover Edition. Academic Press, San Diego, CA.

Peel, A.L., Zolotukhin, S., Schrimsher, G.W., Muzyczka, N. \& Reier, P.J. (1997) Efficient transduction of green fluorescent protein in spinal cord neurons using adeno-associated virus vectors containing cell type-specific promoters. Gene Ther., 4, 16-24.

Peng, S., Wuu, J., Mufson, E.J. \& Fahnestock, M. (2004) Increased proNGF levels in subjects with mild cognitive impairment and mild Alzheimer disease. J. Neuropathol. Exp. Neurol., 63, 641-649.

Pongrac, J.L. \& Rylett, R.J. (1998) NGF-induction of the expression of ChAT mRNA in PC12 cells and primary cultures of embryonic rat basal forebrain. Brain Res. Mol. Brain Res., 62, 25-34.

Reynolds, A., Leake, D., Boese, Q., Scaringe, S., Marshall, W.S. \& Khvorova, A. (2004) Rational siRNA design for RNA interference. Nat. Biotechnol., 22, 326-330.

Roux, P.P. \& Barker, P.A. (2002) Neurotrophin signaling through the p75 neurotrophin receptor. Prog. Neurobiol., 67, 203-233.

Sanchez-Ortiz, E., Yui, D., Song, D., Li, Y., Rubenstein, L., Reichardt, F. \& Parada, L.F. (2012) TrkA gene ablation in basal forebrain results in dysfunction of the cholinergic circuitry. J. Neurosci., 32, 4065-4079.

Saragovi, H.U. (2005) Progression of age-associated cognitive impairment correlates with quantitative and qualitative loss of TrkA receptor protein in nucleus basalis and cortex. J. Neurochem., 95, 1472-1480.

Sarter, M. \& Bruno, J.P. (1998) Age-related changes in rodent cortical acetylcholine and cognition: main effects of age versus age as an intervening variable. Brain Res. Brain Res. Rev., 27, 143-156.
Sarter, M. \& Bruno, J.P. (2004) Developmental origins of the age-related decline in cortical cholinergic function and associated cognitive abilities. Neurobiol. Aging, 25, 1127-1139.

Sarter, M. \& Parikh, V. (2005) Choline transporters, cholinergic transmission and cognition. Nat. Rev. Neurosci., 6, 48-56.

Schor, N.F. (2005) The p75 neurotrophin receptor in human development and disease. Prog. Neurobiol., 77, 201-214.

Sobottka, B., Reinhardt, D., Brockhaus, M., Jacobsen, H. \& Metzger, F. (2008) ProNGF inhibits NGF-mediated TrkA activation in PC12 cells. $J$. Neurochem., 107, 1294-1303.

Sobreviela, T., Clary, D.O., Reichardt, L.F., Brandabur, M.M., Kordower, J.H. \& Mufson, E.J. (1994) TrkA-immunoreactive profiles in the central nervous system: colocalization with neurons containing p75 nerve growth factor receptor, choline acetyltransferase, and serotonin. J. Comp. Neurol., 350, 587-611.

Sofroniew, M.V., Howe, C.L. \& Mobley, W.C. (2001) Nerve growth factor signaling, neuroprotection, and neural repair. Ann. Rev. Neurosci., 24, 1217-1281.

St Peters, M., Demeter, E., Lustig, C., Bruno, J.P. \& Sarter, M. (2011) Enhanced control of attention by stimulating mesolimbic-corticopetal cholinergic circuitry. J. Neurosci., 31, 9760-9771.

Szutowicz, A., Madziar, B., Pawełczyk, T., Tomaszewicz, M. \& Bielarczyk, H. (2004) Effects of NGF on acetylcholine, acetyl-CoA metabolism, and viability of differentiated and non-differentiated cholinergic neuroblastoma cells. J. Neurochem., 90, 952-961.

Taylor, J., Chung, K.H., Figueroa, C., Zurawski, J., Dickson, H.M., Brace, E.J., Avery, A.W., Turner, D.L. \& Vojtek, A.B. (2008) The scaffold protein POSH regulates axon outgrowth. Mol. Biol. Cell, 19, 51815192.

Terry, A.V., Kutiyanawalla, A. \& Pillai, A. (2011) Age-dependent alterations in nerve growth factor (NGF)-related proteins, sortilin, and learning and memory in rats. Physiol. Behav., 102, 149-157.

Verbeke, G. \& Molenberghs, G. (2009) Linear Mixed Models for Longitudinal Data. Springer, New York.

Volosin, M., Song, W., Almeida, R.D., Kaplan, D.R., Hempstead, B.L. \& Friedman, W.J. (2006) Interaction of survival and death signaling in basal forebrain neurons: roles of neurotrophins and proneurotrophins. J. Neurosci., 26, 7756-7766.

West, M.J., Slomianka, L. \& Gundersen, H.J. (1991) Unbiased stereological estimation of the total number of neurons in the subdivisions of the rat hippocampus using the optical fractionator. Anat. Rec., 231, 482-497. 